\title{
Obliquity evolution of extrasolar terrestrial planets
}

\author{
Keiko Atobe and Shigeru Ida \\ E-mail: ida@geo.titech.ac.jp \\ Department of Earth and Planetary Sciences, Tokyo Institute of Technology, \\ 2-12-1 Ookayama, Meguro-ku, Tokyo 152-8551, Japan
}

Icarus, in press

\section{ABSTRACT}

We have investigated the obliquity evolution of terrestrial planets in habitable zones (at $\sim$ $1 \mathrm{AU})$ in extrasolar planetary systems, due to tidal interactions with their satellite and host star with wide varieties of satellite-to-planet mass ratio $\left(m / M_{\mathrm{p}}\right)$ and initial obliquity $\left(\gamma_{0}\right)$, through numerical calculations and analytical arguments. The obliquity, the angle between planetary spin axis and its orbit normal, of a terrestrial planet is one of the key factors in determining the planetary surface environments. A recent scenario of terrestrial planet accretion implies that giant impacts of Mars-sized or larger bodies determine the planetary spin and form satellites. Since the giant impacts would be isotropic, tilted spins $\left(\sin \gamma_{0} \sim 1\right)$ are more likely to be produced than straight ones $\left(\sin \gamma_{0} \sim 0\right)$. The ratio $m / M_{\mathrm{p}}$ is dependent on the impact parameters and impactors' mass. However, most of previous studies on tidal evolution of the planet-satellite systems have focused on a particular case of the Earth-Moon systems in which $m / M_{\mathrm{p}} \simeq 0.0125$ and $\gamma_{0} \sim 10^{\circ}$ or the two-body planar problem in which $\gamma_{0}=0^{\circ}$ and stellar torque is neglected. We numerically integrated the evolution of planetary spin and a satellite orbit with various $m / M_{\mathrm{p}}$ (from 0.0025 to 0.05 ) and $\gamma_{0}$ (from $0^{\circ}$ to $180^{\circ}$ ), taking into account the stellar torques and precessional motions of the spin and the orbit. We start with the spin axis that almost coincides with the satellite orbit normal, assuming that the spin and the satellite are formed by one dominant impact. With initially straight spins, the evolution is similar to that of the Earth-Moon system. The satellite monotonically recedes from the planet until synchronous state between the spin period and the satellite orbital period is realized. The obliquity gradually increases initially but it starts decreasing down to zero as approaching the synchronous state. However, we have found that the evolution with initially tiled spins is completely different. The satellite's orbit migrates outward with almost constant obliquity until the orbit reaches the critical radius $\sim 10-20$ planetary radii, but then the migration is reversed to inward one. At the reversal, the obliquity starts oscillation with large amplitude. The oscillation gradually ceases and the obliquity is reduced to $\sim 0^{\circ}$ during the inward migration. The satellite eventually falls onto the planetary surface or it is captured at the synchronous state at several planetary radii. We found that the character change of precession about total angular momentum vector into that about the planetary orbit normal is responsible for the oscillation with large amplitude and the reversal of migration. With the results of numerical integration and analytical arguments, we divided the $m / M_{\mathrm{p}}-\gamma_{0}$ space into the regions of the 
qualitatively different evolution. The peculiar tidal evolution with initially tiled spins give deep insights into dynamics of extrasolar planet-satellite systems and discussions of surface environments of the planets.

Key Words: Extrasolar planets - Satellites, dynamics - Celestial mechanics - Rotational dynamics - Tide, solid body

\section{Introduction}

Recently, extrasolar planets of $5-20 M_{\oplus}$, which may be rocky/icy planets, have started being discovered by development of radial velocity survey (e.g., Butler et al. 2004, Rivera et al. 2005, Lovis et al. 2006) and gravitational microlensing survey (Beaulieu et al. 2006), although the majority of extrasolar planets so far discovered have masses larger than Saturn's mass $\left(\gtrsim 100 M_{\oplus}\right)$, which may be gas giant planets. If the core accretion model (e.g., Mizuno 1980; Bodenheimer and Pollack 1986) is responsible for formation of the extrasolar gas giants, their high occurrence rate $(\gtrsim 5 \%)$ implies the ubiquity of extrasolar terrestrial planets (e.g., Ida and Lin 2004), because cores of gas giants are formed through planetesimal accretion and failed bodies that are not massive enough for gas accretion onto them are no other than terrestrial planets and icy planets. Near-future space telescopes, COROT and KEPLER may find Earthsize planets, through transit survey, including those within so-called "habitable zones," where planets can maintain liquid water on their surfaces (e.g., Borucki et al. 2003; Ruden 1999).

In addition to existence of liquid water, stable climate on timescales more than $10^{9}$ yrs may be one of essential components for planets to be habitable, in particular, for a land-based life. Planetary global climate is greatly influenced by insolation distribution (Milankovitch 1941; Berger 1984, 1989), which is largely related with obliquity $\gamma$, the angle between the spin axis and the orbit normal of the planet (Ward 1974). For example, if $\gamma>54^{\circ}$, the planet receives more annual-averaged insolation at the poles than at the equator and vice versa. For high $\gamma$, seasonal cycles at high latitudes would also become very pronounced (Williams and Kasting 1997). Abe et al. (2005) indicated that obliquity is a very important factor for atmospheric transport of water to low-latitude areas.

Planetary obliquity evolves mainly by tidal interactions with its satellite and host star. All of the terrestrial planets in our solar system do not maintain their primordial spin state (obliquity $\gamma$ and spin frequency $\Omega$ ). Mercury spins with $\gamma=0$ and $\Omega$ of precisely $3 / 2$ times as large as its orbital mean motion (Colombo 1965; Colombo and Shapiro 1965). This configuration is an outcome of the tidal interaction with the Sun (e.g., Goldreich 1966; Goldreich and Peale 1966). Venus rotates slowly with a 243-day period and $\gamma \sim 180^{\circ}$. This spin state could be an equilibrium between gravitational and thermal atmospheric tidal torque (Gold and Soter 1969) or an outcome of friction at a core-mantle boundary (Goldreich and Peale 1970). Dissipation effects combined with planetary perturbations could bring the spin axis to $180^{\circ}$ from any initial $\gamma$ (Nérson de Surgy 1996; Yorder 1997; Correia and Laskar 2001).

The Earth's obliquity is gradually increasing with the receding of the Moon as a consequence of the tidal dissipation in Earth mainly induced by the Moon (e.g., Darwin 1879; Goldreich 1966). Figures 1 show the evolutionary path of the Earth's obliquity $(\gamma)$ and orbital inclinations of the Moon's orbit to the ecliptic $(i)$ and the Earth's equator $(\epsilon)$, which is obtained by integrating the present Earth-Moon system back into the past (for integration method, see

section 2). The ranges of oscillation during precession are indicated by shaded regions. This plots will be refereed to later. 
[Figure 1]

Mars' obliquity would be suffering from a large-scale oscillation of $\sim 25^{\circ} \pm 10^{\circ}$ on a timescale $\sim 10^{5}-10^{6}$ years (Ward 1973, 1974, 1979), by a resonance between a spin precession rate and one of the eigenfrequencies of its orbital precession. The spin-orbit resonance is a different mechanism to alter planetary spin state, from the tidal evolution. Because Earth's spin precession is accelerated out of the resonance by the Moon, Earth's current obliquity fluctuates with only $\pm 1.3^{\circ}$ around $23.3^{\circ}$ (Ward 1974; Laskar and Robutel 1993; Laskar 1996).

Here, we focus on evolution of planet's spin state and its satellite orbit due to the tidal dissipation in the planet caused by the satellite and the host star. The spin-orbit resonance in extrasolar planetary systems was addressed in detail elsewhere (Atobe et al. 2004) and will be commented on in section 4. When the tide raising body (star and/or satellite) orbits around the spinning planet, the planet is deformed by the tide with a certain time interval, resulting in a lag angle $\delta$ as illustrated in Fig. 2. ( $\delta$ can be either positive or negative.) The attraction of the tide raising body yields a torque on the planet and an equal but opposing torque on the body. In the case of Fig. 2, the torque retards the planet spin and increases the orbital angular momentum and hence semi-major axis of the tide raising body. If the equatorial plane of the planet does not coincide with the orbital plane of the tide raising body, this angular momentum exchange also changes the obliquity of the planet and the orbital inclination of the tide raising body. The tidal torque depends on the mass of the tide raising body and strongly on the separation between the bodies. The tide raised on Mercury and Venus are due to the Sun, while that on Earth is mainly induced by the Moon rather than the Sun.

[Figure 2]

As we will show in later sections, obliquity evolution at $\sim 1 \mathrm{AU}$ is regulated by a satellite if the satellite-to-planet mass ratio is $\gtrsim 0.01$. The Moon would have been formed by a grazing collision with a Mars-mass object during the late stage of Earth accretion (e.g., Stevenson 1987, Canup 2004). Oligarchic growth model (Kokubo and Ida 1998, 2000) predicts formation of isolated Mars-mass bodies at $\sim 1 \mathrm{AU}$ in the case of the minimum mass solar nebula (Hayashi 1981). The isolated bodies would start orbit crossing by long term distant perturbations on timescales longer than than Myrs (Chambers et al. 1996, Iwasaki et al. 2002). Thus, giant impacts with objects of more than Mars-mass would be common and satellites may be produced if the impacts are grazing ones. The satellite mass is determined by total mass and angular momentum of the impact-debris disk (Ida et al. 1997; Kokubo et al. 2000), which would be regulated by the impact parameter of the collision and the impactor's mass.

If planetary spin is mostly determined by the giant impact forming a satellite (Lissauer et al. 2000), the spin axis would align with the orbit normal of the satellite. Recent N-body simulations of the planet accretion show that satellite forming impacts are almost isotropic (Agnor et al. 1999; Chambers 2001). Hence it is expected that the primordial obliquity $\gamma_{0}$ has the differential distribution, $p\left(\gamma_{0}\right) d \gamma_{0}=\frac{1}{2} \sin \gamma_{0} d \gamma_{0}$, so that the most common initial spins are tilted ones $\left(\gamma_{0} \sim 90^{\circ}\right)$. As we will show, the obliquity evolution would be quite different in cases of tilted initial spins from the familiar evolution in Fig. 1. In order to investigate obliquity evolution of extrasolar terrestrial planets, evolution with wide ranges of initial obliquity $\left(\gamma_{0}=\right.$ $0^{\circ}-180^{\circ}$ ) and satellite mass should be studied. Many studies on obliquity evolution have been focusing on the Earth-Moon system of $\gamma_{0} \sim 10^{\circ}$. Although Counselman (1973) and Ward and Reid (1973) addressed more general characteristics and outcome of tidal evolution, they assumed that the mutual inclinations among the satellite's orbit, planet's equator and orbit are always 
zero, i.e., $\gamma=0$ (for details of Counselman (1973), see section 3.2). However, as mentioned above, the planetary spin of terrestrial planets is more likely to be initially perpendicular to its orbit normal $\left(\gamma_{0} \sim 90^{\circ}\right)$.

In this study, we investigate the tidal evolution of planet-satellite systems with wide ranges of satellite-to-planet mass ratio and initial obliquity, numerically calculating the planet's spin state and satellite's orbit. We will show that character change of precessional motions during tidal evolution plays an important role in producing diversity of the tidal evolution. In Section 2, we describe basic equations for precession and tidal evolution. The initial conditions are also described. Section 3 describes the results of the simulations and general features of tidal evolution. Section 4 and 5 are devoted to discussion and conclusion.

\section{Model and the basic equations}

\section{$2.1 \quad$ Model}

We consider a three-body system composed of a host star with mass $M_{*}$, a planet with mass $M_{\mathrm{p}}$ and physical radius $R_{\mathrm{p}}$, and a satellite with mass $m$. For simplicity, we assume that the planet-satellite system is in the circular orbit around the host star with the mean motion $n_{\mathrm{p}}$ and that the satellite is in the circular orbit around the planet with the mean motion $n$.

We adopted the planet-centric frame $(X, Y, Z)$. In this frame, the satellite and the central star rotate around the planet with $n$ and $n_{\mathrm{p}}$, respectively. Figure 3 shows the geometry of the orbital and equatorial planes of the planet, and the orbital plane of the satellite. $\mathbf{s}, \mathbf{k}$, and $\mathbf{n}$ represent unit vectors in the directions of spin axis of the planet, its orbit normal, and the satellite orbit normal, respectively. In this frame, $\mathbf{k}$ is a fixed unit vector in the $Z$-direction. (Although the frame with $\mathbf{n}$ fixed might be better to discuss the issue of diversity of tidal evolution, we adopt the frame that is more intuitive.) We denote the obliquity of the planet (the angle between $\mathbf{k}$ and $\mathbf{s}$ ) by $\gamma$, the inclination of the satellite orbit to the planet orbit (the angle between $\mathbf{k}$ and $\mathbf{n}$ ) by $i$, and that to the planetary equator (the angle between $\mathbf{n}$ and $\mathbf{s}$ ) by $\epsilon$. We assume the planet as an axisymmetric fluid rotating with geometrical axis always parallel to its spin angular momentum vector. The planetary spin angular velocity is denoted by $\Omega$. The satellite spin angular momentum is neglected (Appendix B).

[Figure 3]

\subsection{Basic equations}

The scalar angular momentum of the planetary spin and that of the satellite orbital motion are denoted by $H$ and $h$. Denoting the total precessional torques acting on the planet and the satellite by $\mathbf{L}_{p}$ and $\mathbf{L}_{\mathrm{s}}$ and the tidal torques by $\mathbf{T}_{\mathrm{p}}$ and $\mathbf{T}_{\mathrm{s}}$, the equations of motion of the planetary spin and the satellite orbit are

$$
\begin{aligned}
\frac{d H \mathbf{s}}{d t} & =\mathbf{L}_{\mathrm{p}}+\mathbf{T}_{\mathrm{p}}, \\
\frac{d h \mathbf{n}}{d t} & =\mathbf{L}_{\mathrm{s}}+\mathbf{T}_{\mathbf{s}} .
\end{aligned}
$$

The precessional torques just rotate the direction of $\mathbf{s}$ and $\mathbf{n}$, keeping $H$ and $h$ constant (Eqs. (39) and (40) in Appendix A). The tidal torques alter $H$ and $h$. Since angular momentum 
is also exchanged with the planetary orbit, $(H \mathbf{s}+h \mathbf{n})$ does not conserve. But, since the planet orbital angular momentum is much larger than $H$ and $h$, we assume that $\mathbf{k}$ is invariant.

We adopt the method by Goldreich (1966) to calculate Eqs. (1) and (2) because it follows precessional motions, although some formalisms (e.g., Mignard 1978, 1979, 1980) are averaged over the precession. The precessional motions play a key role in the tidal evolution of the system in the case of high initial $\gamma$. (For the Earth-Moon system with relatively low initial $\gamma$, predicted tidal evolution is hardly affected by whether precessional motions are taken into account or not.) Goldreich's method is a multiple averaged "secular theory," utilizing three distinct timescales: orbital periods, precessional ones, and tidal friction timescale. Because tidal evolution timescale is usually much longer than precession periods, $\mathbf{T}_{\mathrm{p}}$ and $\mathbf{T}_{\mathrm{s}}$ are neglected in the equations to describe precessional motions. The equations are (Goldreich 1966; also see Appendix A)

$$
\begin{aligned}
H \frac{d \mathbf{s}}{d t} & \simeq \mathbf{L}_{\mathrm{p}}=L(\mathbf{s} \cdot \mathbf{n})(\mathbf{s} \times \mathbf{n})+K_{1}(\mathbf{s} \cdot \mathbf{k})(\mathbf{s} \times \mathbf{k}) \\
h \frac{d \mathbf{n}}{d t} & \simeq \mathbf{L}_{\mathrm{s}}=-L(\mathbf{s} \cdot \mathbf{n})(\mathbf{s} \times \mathbf{n})+K_{2}(\mathbf{n} \cdot \mathbf{k})(\mathbf{n} \times \mathbf{k}),
\end{aligned}
$$

where all the quantities are averaged over the orbital periods (the shortest timescales) of the star and the satellite around the planet. $H$ and $h$ are constant with time on the precessional periods (Appendix A). The first terms in r. h. s. of Eqs. (3) and (4) express the precessional torques between the planet and satellite that cause precession around $H \mathbf{s}+h \mathbf{n}$, and the second ones the stellar torques that cause the precession around $\mathbf{k}$. The constants $L, K_{1}$, and $K_{2}$ and detailed expressions of Eqs. (3) and (4) for numerical integration are given in Appendix A. In addition to $H$ and $h, \Lambda_{Z}=H x+h y$ (the $Z$-component of total angular momentum in the planet-satellite system) and $\chi=K_{1} x^{2}+K_{2} y^{2}+L z^{2}$ (a kind of total potential energy) are also conserved (Appendix A), where $x=\mathbf{s} \cdot \mathbf{k}=\cos \gamma, y=\mathbf{n} \cdot \mathbf{k}=\cos i$, and $z=\mathbf{s} \cdot \mathbf{n}=\cos \epsilon$.

The tidal evolution is

$$
\frac{d H \mathbf{s}}{d t} \simeq \mathbf{T}_{\mathrm{p}} ; \quad \frac{d h \mathbf{n}}{d t} \simeq \mathbf{T}_{\mathrm{s}}
$$

where $\mathbf{s}$ and $\mathbf{n}$ are averaged over precession periods (while those in Eqs. (3) and (4) are instantaneous ones), and $\mathbf{L}_{\mathrm{p}}$ and $\mathbf{L}_{\mathrm{s}}$ vanish by the precession averaging. $\mathbf{T}_{\mathrm{p}}$ and $\mathbf{T}_{\mathrm{s}}$ are numerically averaged over the precession periods. We only consider planetary tides induced by the star and the satellite (Appendix B). When the tidal torques are included, $H, h, \Lambda_{Z}, \chi$, and $a$ that are treated as constant in Eqs. (3) and (44) change slowly with time (Eqs. 48, 51, 52, 54, in Appendix A). We adopt the constant time lag model by Mignard (1981) and Touma and Wisdom (1994) for $\mathbf{T}_{\mathrm{p}}$ and $\mathbf{T}_{\mathrm{s}}$ (Appendix $\mathrm{C}$ ).

\subsection{Initial conditions}

If the initial planetary spin state is determined by the satellite forming impact, resulting obliquity would have the distribution stated in Introduction section and the initial spin period would be a few hours if perfect accretion is assumed (Agnor et al. 1999; Chambers 2001). N-body simulations of the accumulation of a satellite in a circumplanetary disk predict that the satellite is formed in an orbital plane close to the planet's equatorial plane $(\epsilon \sim 0)$ at an orbital radius 2.6-4.6R (Ida et al. 1997; Kokubo et al. 2000). The mass of the formed satellite depends on an impact parameter and the mass of a projectile and a target.

We simulated the tidal evolution and the evolutionary path of the obliquity and the satellite orbit with various $m / M_{\mathrm{p}}$ and initial obliquity $\gamma_{0}: m / M_{\mathrm{p}}=0.0025 \times j(j=1,2, \cdots 26), \gamma_{0}=$ 
$10 \times l(l=1,2, \cdots 8,10, \cdots 17)$. In the numerical calculations, the initial semi-major axis of the satellite, $a_{0}$, and the initial rotation period, $D_{0}$, were chosen to be $3.8 R_{\mathrm{p}}$ and 5 hours. Although the simulation is limited to these $a_{0}$ and $D_{0}$, we will present the dependence of the results on $a_{0}$ and $D_{0}$ through analytical arguments. As shown later, key quantities to regulate diversity of tidal evolution are the radius of precession-type change $\left(a_{\text {crit }}\right)$ and the outer co-rotation radius $\left(a_{\mathrm{c}, \text { out }}\right)$. Although $a_{\mathrm{c} \text {,out }} \propto D_{0}^{-2}$ (Eq. 27),$a_{\text {crit }}$ is dependent on $a_{0}$ and $D_{0}$ only weakly $\left(a_{0}\right.$ does not affect $a_{\mathrm{c}, \text { out }}$ either). As long as $D_{0}$ and $a_{0}$ differ from the above values within a factor of a few, the overall features of the results presented here do not change. Initial inclination of the satellite orbit to the planet's equator, $\epsilon_{0}$, is assumed to $1^{\circ}$. We also carried out the calculation with $\epsilon_{0}=5^{\circ}$ and $10^{\circ}$ (results are not shown in this paper). Increase in $\epsilon_{0}$ results in an only slight increase in the asymptotic value of $i$ at large $a$ and corresponding oscillation of $\epsilon$ due to precession (compare Fig. 1 with Fig. 5). However, other properties of evolution are similar. If evolution in the case of Fig. 1 is continued, $\gamma$ and $\epsilon$ will start decreasing to zero as approaching the co-rotation radius. On the assumption that satellites are formed by a giant impact, $\epsilon_{0}$ would not take larger values. Other parameters are assumed that $M_{*}=1 M_{\odot}, a_{\mathrm{p}}=1 \mathrm{AU}$, $M_{\mathrm{p}}=1 M_{\oplus}, \alpha=0.33, \rho=5.5 \mathrm{gcm}^{-3}, k_{s}=0.95, k_{2}=0.30$, and the constant time lag $\delta t=11.5$ min. The dependences of the results on these parameters will be also presented. We integrate the evolution until the synchronous state is achieved or the satellite orbit decays to $2 R_{\mathrm{p}}$. In the latter case, we regard that the satellite falls onto the planet.

\subsection{Precessional motions}

In order to understand the numerical results of tidal evolution, we briefly summarize characteristic behaviors of precessional motions. Three different types of precessional motions are shown in Figs. 4, which were obtained by numerical integration. The trajectories of $\mathbf{s}$ and $\mathbf{n}$ are projected onto the planetary orbital plane $(X, Y)$, in the Earth-Moon system, for (a) $a \simeq 5 R_{\oplus}$, (b) $a \simeq 15 R_{\oplus}$, and (c) $a \simeq 60 R_{\oplus}$ (the present location), respectively. Although these different precessional motions were already discussed in detail by Goldreich (1966) and Touma \& Wisdom (1994), we present the summary again, because the character changes in precessional motion play an important role to produce diversity of obliquity evolution.

When $a \simeq 5 R_{\oplus}$, the interaction between the Earth and the Moon is much greater than their interaction with the Sun, i.e., $L \gg K_{1}, K_{2}$ in Eqs. (3) and (4). In this case, s (denoted by a solid curve and filled squares) and $\mathbf{n}$ (denoted by a dashed curve and filled triangles) precess about a common axis with the same precession speeds, resulting in constant $\epsilon$, the angle between $\mathbf{s}$ and $\mathbf{n}$. The common axis precesses slowly about $\mathbf{k}((X, Y)=(0,0))$ by the stellar torques, as indicated in Fig. 4 a. Hereafter, we call this motion type I precession. When $a \simeq 15 R_{\oplus}$, torques on the Moon's orbit due to the Earth and the Sun are nearly equal, on the other hand, those on the Earth is mainly due to the Moon, i.e., $K_{2} \sim L>K_{1}$. In this case, $\mathbf{n}$ precesses about the average position of $\mathbf{s}$ and $\mathbf{k}$, while $\mathbf{s}$ tends to precess about $\mathbf{n}$ and is dragged by the motion of $\mathbf{n}$, as illustrated in Fig. 4b. We call this motion type II precession. In the current state, the Sun's torque on the Moon's orbit is much larger than the Earth's torque, while the Moon's torque on the Earth is about 2 times larger than the Sun's torque, i.e., $K_{2} \gg L>K_{1}$. In this case, $\mathbf{n}$ precesses about $\mathbf{k}$ maintaining $i$ almost constant. Although $\mathbf{s}$ still tends to precesses about $\mathbf{n}, \mathbf{s}$ actually precesses about $\mathbf{k}$ because $\mathbf{n}$ precesses about $\mathbf{k}$ on much shorter period (18 years) than the precession period (27000 years) of $\mathbf{s}$ about $\mathbf{n}$, as illustrated in Fig. 4k. We call this motion type III precession.

[Figure 4] 
We define the critical orbital radius of the satellite, $a_{\text {crit }}$, by $K_{2}=L$, at which type I precession is transformed into type II. From Eqs. (35) and (38),

$$
\frac{K_{2}}{L}=\frac{3}{2} \frac{G M_{*}}{k_{\mathrm{s}} \Omega^{2} a_{\mathrm{p}}^{3}}\left(\frac{a}{R_{\mathrm{p}}}\right)^{5}=\frac{3}{2 k_{\mathrm{s}}}\left(\frac{n_{\mathrm{p}}}{\Omega}\right)^{2}\left(\frac{a}{R_{\mathrm{p}}}\right)^{5},
$$

so that $a_{\text {crit }}$ is given by

$$
\frac{a_{\text {crit }}}{R_{\mathrm{p}}}=\left(\frac{2 k_{\mathrm{s}}}{3}\right)^{1 / 5}\left(\frac{\Omega}{n_{\mathrm{p}}}\right)^{2 / 5}=18.5 k_{\mathrm{s}}^{1 / 5}\left(\frac{M_{*}}{1 M_{\odot}}\right)^{-1 / 5}\left(\frac{a_{\mathrm{p}}}{1 \mathrm{AU}}\right)^{3 / 5}\left(\frac{D}{5 \mathrm{hrs}}\right)^{-2 / 5},
$$

where $D$ is the spin period of the planet $(=2 \pi / \Omega)$ at $a_{\text {crit. }}$. For the Earth, $a_{\text {crit }} \simeq 17 R_{\oplus}$ (Goldreich 1966). Although $D$ can take various values according to tidal evolution from $D_{0}$, the dependence on $D$ is weak.

Because the ratio $K_{2} / L$ varies as the fifth power of $\left(a / R_{\mathrm{p}}\right)$, the precession of the satellite is almost completely dominated by the planetary torque (type I precession) when $a<a_{\text {crit }}$ and by the stellar torque (type III precession) when $a>a_{\text {crit }}$ (Goldreich 1966). Type II precession near $a_{\text {crit }}$ results in changes in all of $\gamma, i$, and $\epsilon$. In the case of initially tilted spins, this fluctuation is so large that outward migration of the satellite orbit is transformed into inward one (see section $3)$.

\section{Results of the tidal evolution}

\subsection{Diversity of tidal evolution}

Our numerical calculations show qualitatively different three types (A, B, C) of tidal evolution: A) the satellite orbit monotonically expands outward until $a$ reaches an outer co-rotation radius, B) it expands to $\sim a_{\text {crit }}$ and turns back onto the planet, and C) the same as B) but it is locked at a synchronous state before falling onto the planet. The tidal evolution of the Earth-Moon system belongs to type A evolution, so that characteristics of type A evolution have been well known. On the other hand, type $\mathrm{B}$ and $\mathrm{C}$ evolution is newly found by us. The evolution is type $\mathrm{B}$ or $\mathrm{C}$ if an initial spin is tilted, as shown below. Since tilted initial spins are most common, as we stated in Introduction section, the newly found type $\mathrm{B}$ and $\mathrm{C}$ evolution would be more common than type A for extrasolar terrestrial planets.

\subsubsection{Tidal evolution with initially straight spins}

Figures 5 show a typical result of type A evolution with $m=0.02 M_{\mathrm{p}}$ and $\gamma_{0}=10^{\circ}$ : (a) obliquity $\gamma$, (b) $i,(\mathrm{c}) \epsilon$, and (d) the semimajor axis of the satellite $(a)$, the estimated inner and co-rotation radii $\left(a_{\mathrm{c}, \text { in }}\right.$ and $a_{\mathrm{c}, \text { out }}$ ) (see below and section 3.3). $\gamma, i$ and $\epsilon$ are plotted as functions of $a$. The semimajor axis is plotted as a function of time. The evolution in this case is qualitatively similar to that of the Earth-Moon system in Figs. 1.

In the proximity of the planet, the spin axis of the planet (s) and the orbit normal of the satellite $(\mathbf{n})$ precess about the common axis with nearly constant mutual inclination $\epsilon$ (type I precession). As long as $a \lesssim a_{\text {crit }} \sim 15 R_{\mathrm{p}}$, the satellite orbit migrates outward maintaining $\epsilon \sim 0$ (an initial value) and accordingly $\gamma \sim i$. As the satellite approaches $a_{\text {crit }}, \mathbf{n}$ tends to precess about the average position of $\mathbf{s}$ and $\mathbf{k}$ (type II precession). Because of this transition of precession, $\mathbf{s}$ and $\mathbf{n}$ begin to precess at different rates so that $\epsilon$ increases. 
When $a>a_{\text {crit }}$, $\mathbf{s}$ and $\mathbf{n}$ precess about $\mathbf{k}$ with different speeds (type III precession). $i$ quickly decreases to zero, while $\gamma$ starts increasing (Fig. 5). These behaviors are explained as follows. From Eqs. (49) and (50) in Appendix A with tidal torque formula in Appendix C,

$$
\begin{aligned}
\frac{d x}{d t} & =-\frac{3}{2} \frac{G m^{2} R_{\mathrm{p}}^{5} k_{2} \delta t}{a^{6}} \frac{(y-x z)}{H}(\Omega z-2 n)-\frac{3}{2} \frac{G M_{*}^{2} R_{\mathrm{p}}^{5} k_{2} \delta t}{a_{\mathrm{p}}^{6}} \frac{\left(1-x^{2}\right)}{H}\left(\Omega x-2 n_{\mathrm{p}}\right), \\
\frac{d y}{d t} & =\frac{3}{2} \frac{G m^{2} R_{\mathrm{p}}^{5} k_{2} \delta t}{a^{6}} \frac{(x-y z)}{h} \Omega .
\end{aligned}
$$

where $x=\mathbf{s} \cdot \mathbf{k}=\cos \gamma, y=\mathbf{n} \cdot \mathbf{k}=\cos i, z=\mathbf{s} \cdot \mathbf{n}=\cos \epsilon$, and $\delta t$ is a time lag for distortion of the planet (Appendix C). In Eq. (8), the first and the second terms express the stellar and satellite's tidal torques. In this region, the satellite tide is dominant. Neglecting the second term, $d x / d t<0$ if $\Omega z>2 n$, because usually $y>x z$ in this regime. Since $\Omega \gg n$ except for late phase near the co-rotation radius, $\gamma$ increases toward the asymptotic value of $\gamma, \cos ^{-1}(2 n / \Omega)$, which is $\sim 90^{\circ}$ for $\Omega z / n \gg 2$. Equation (9) includes only a stellar torque. Since usually $x>y z$ in this regime, $d y / d t>0$ and the stellar torque decreases $i$ to zero throughout the tidal evolution.

From Eqs. (53) and (54) with tidal torque formula in Appendix C, evolution of the spin frequency $\Omega$ and the satellite semimajor axis $a$ is

$$
\begin{aligned}
& \frac{d \Omega}{d t}=-\frac{3}{2} \frac{G m^{2} R_{\mathrm{p}}^{5} k_{2} \delta t}{a^{6}} \frac{1}{I_{\mathrm{p}}}\left\{\Omega\left(1+z^{2}\right)-2 n z\right\} \\
&-\frac{3}{2} \frac{G M_{*}^{2} R_{\mathrm{p}}^{5} k_{2} \delta t}{a_{\mathrm{p}}^{6}} \frac{1}{I_{\mathrm{p}}}\left\{\Omega\left(1+x^{2}\right)-2 n_{\mathrm{p}} x\right\}, \\
& \frac{d a}{d t}=\frac{3}{2} \frac{G m^{2} R_{\mathrm{p}}^{5} k_{2} \delta t}{a^{6}} \frac{4 a}{h}(\Omega z-n),
\end{aligned}
$$

where $I_{\mathrm{p}}$ is the planet's moment of inertia, given by $\alpha M_{\mathrm{p}} R_{\mathrm{p}}^{2}$ (where $\alpha \leq 2 / 5$; for the Earth, $\alpha \simeq$ 0.33). Neglecting the stellar torque (the second term) in Eq. (10) (assuming $m^{2} / a^{6} \gg M_{*}^{2} / a_{\mathrm{p}}^{6}$ ), $d \Omega / d t<0$ if $\Omega>\left(2 \cos \epsilon /\left(1+\cos ^{2} \epsilon\right)\right) n$. Thus, as long as $\Omega>2 n$, the spin rate $\Omega$ decreases for any value of $\epsilon$. Eventually $\Omega \cos \epsilon$ becomes smaller than $2 n$, so that $d x / d t>0$ (Eq. 8) and $\gamma$ begins to decrease, as shown in Fig. 5r. The asymptotic value is $\cos ^{-1}(2 n / \Omega) \rightarrow 0^{\circ}$ as $\Omega z / n \rightarrow 2$. For $\Omega z / n<2, \gamma$ approaches $0^{\circ}$ regardless of its value.

This decrease in $\gamma$ causes the stable synchronism $(\Omega \sim n)$ with $\gamma \sim i \sim \epsilon \sim 0$. Equation (11) indicates the satellite is receding from the planet when $\Omega z / n>1$. The co-rotation radius $a_{\mathrm{c}}$ at which $\Omega z / n=1$ depends on the value of $z$. There are generally two co-rotation radii in the prograde case; the synchronous state is stable at outer one $a_{\mathrm{c}, \text { out }}$ while it is unstable at inner one $a_{\mathrm{c}, \text { in }}$ (section 3.2). At $a>a_{\text {crit }}$ in type A evolution here, $z>0$, so that $a$ monotonically increases. As $a \rightarrow a_{\mathrm{c} \text {,out }}$, the orbital expansion of the satellite terminates $(d a / d t=0)$. As discussed in the above, $\gamma \sim i \sim 0$ (and hence, $\epsilon \sim 0$ ) as approaching $a_{\mathrm{c}, \text { out }}$, so that $a_{\mathrm{c}, \text { out }}$ is given by $\Omega=n$ in this case.

Note that the weak stellar tidal torque secularly decreases the planetary spin and hence $a_{\mathrm{c}, \text { out }}$ (Eq. 10). As a result, the satellite orbit slowly decays, being locked at $a_{\mathrm{c}, \text { out }}$ (Ward and Reid 1973), although the decay timescale is longer than $10^{10}$ years for a planet at $1 \mathrm{AU}$ (e.g., Goldreich 1966; also see Eq. 34).

\subsubsection{The cases of initially tilted spins}

[Figure 6] 
Figures 6 show the evolution in the case of $m=0.01 M_{\mathrm{p}}$ and $\gamma_{0}=80^{\circ}$. During type I precession up to $\sim 18 R_{\mathrm{p}}, \gamma$ and $i$ are kept almost constant. At $a \sim 18 R_{\mathrm{p}}$, type II precession starts. As a consequence of transition from type I to type II precession, $\epsilon$ suddenly starts oscillation with a large amplitude (Fig. 6r). During type I precession, since $\epsilon_{0}=1^{\circ}$ and $\epsilon$ is almost conserved, $\mathbf{s}$ and $\mathbf{n}$ always point to the same direction as illustrated in the left panel of Fig. 7. In the case of type III precession, $\mathbf{s}$ and $\mathbf{n}$ tend to precess about $\mathbf{k}$ with different frequencies. If type III precession starts with $\gamma_{0}$ and $i_{0}, \epsilon \sim \epsilon_{0} \sim 0$ when $\mathbf{s}$ and $\mathbf{n}$ are at the same precession phases, while $\epsilon \sim 2 \gamma_{0}$ when $\mathbf{s}$ and $\mathbf{n}$ are at the opposite precession phases (the right panel of Fig. 7). When precession is transformed to type II precession at $a \sim a_{\text {crit }}, \mathbf{s}$ and $\mathbf{n}$ tend to precess differently, although not completely. Hence, when $a$ becomes $\sim a_{\text {crit }}$, the precession tends to have $\epsilon$ oscillate in the range up to $\sim 2 \gamma_{0}$, which is consistent with the numerical result in Fig. 6r.

[Figure []

Equation (11) shows that the orbital migration is inward when $\Omega z / n=\Omega \cos \epsilon / n<1$. Because of the large fluctuation of $\epsilon$ caused by large $\gamma_{0}, \Omega \cos \epsilon / n<1$ during large part of precession. The satellite orbit migrates back and forth during a precession period. In the case of Fig. 6, the net migration is inward during type II precession. Approaching the planet, the interaction between the planet and satellite dominates the precession again, and $\mathbf{s}$ and $\mathbf{n}$ begin to precess about each other (back to type I precession). The amplitude of precessional oscillation of $\epsilon$ deceases as the satellite orbit comes back to the type I precession regime. The asymptotic value of $\epsilon$ is larger than $90^{\circ}$ in the case of Fig. 6; satellite's orbit becomes retrograde around the planet (in which $a_{\mathrm{c}}$ vanishes). Hence, satellite eventually falls onto the planet.

In section 3.2.2, the reversal of migration at $a \sim a_{\text {crit }}$ is explained in terms of angular momentum and energy and the condition for the reversal is presented. In previous studies, only the stellar tidal torque has been considered for a mechanism for eventual decay of initially prograde satellite orbits. Here we have newly found that planet-satellite tidal interactions can reverse the orbital migration in the case of tilted spins without any help of the stellar tidal torques.

\subsubsection{The cases of initially moderately tilted spins}

Figures 8 show the evolution in the case of $m=0.04 M_{\mathrm{p}}$ and $\gamma_{0}=40^{\circ}$. In this case, $a_{\text {crit }} \sim 14 R_{\mathrm{p}}$. The evolution is similar to that in Fig. 6 until $a$ turns back from $a_{\text {crit }}$. In this case, however, the satellite's orbital and planetary spin periods are locked at stable synchronism. Figure [8d shows the time evolution of $a$ and the co-rotation radii $a_{\mathrm{c}, \text { in }}$ and $a_{\mathrm{c} \text {,out }}$ calculated by $L_{Z 0}=L_{Z \mathrm{c}}$ (Eqs. 23 and 25). Since the co-rotation radii are defined by $\Omega \cos \epsilon=n$, it largely oscillates due to the oscillation of $\epsilon$ on the precession period, at $a \sim a_{\text {crit }}$. In Fig. 8d, such oscillation occurs from $0.4 \times 10^{5}$ years to $2.2 \times 10^{5}$ years. Since the oscillation is so violent, $a_{\mathrm{c}, \text { out }}$ and $a_{\mathrm{c}, \text { in }}$ during this period is omitted in Fig. 8 d. During this period, $a_{\mathrm{c}, \text { out }}$ changes so rapidly that it passes through the satellite orbit without capturing the orbit at the synchronism $(t=0.5-$ $1.0 \times 10^{5}$ years). After the oscillation ceases, $a$ approaches $a_{\mathrm{c}, \text { out }}$ from the outside of $a_{\mathrm{c}, \text { out }}$, and the satellite orbit is captured at $a_{\mathrm{c}, \text { out }}$. The condition for the capture is analytically derived in section 3.2.2. This evolution is also newly found one in the present paper.

[Figure 8] 


\subsubsection{High $m$ cases}

If the satellite is massive $\left(m \gtrsim 0.05 M_{\mathrm{p}}\right)$, the planetary spin and the satellite's orbit become synchronous before the occurrence of the precession transition. In this case, $\gamma$, $i$, and $\epsilon$ are almost conserved as the initial values. We will refer to this type of evolution as type A2 evolution and the evolution in section 3.1.1 as A1.

\subsubsection{Small $m$ cases}

If the satellite is light enough, the stellar tidal torques dominate over the satellite torques. In this case, $\Omega$ becomes smaller than $n$ before the obliquity becomes zero, then the satellite begins to decay toward planet very slowly. The subsequent reduction of the planetary spin leads to a synchronous state with planetary mean motion $\left(\Omega=n_{\mathrm{p}}\right)$ with $\gamma \sim i \sim \epsilon \sim 0$. We will refer to this as type A3 evolution.

\subsubsection{Retrograde cases}

If an initial planetary spin is determined by a satellite forming impact, retrograde spins $\left(\gamma_{0}>\right.$ $\left.90^{\circ}\right)$ have equal probability to prograde spins $\left(\gamma_{0}<90^{\circ}\right)$. Since we assume that $\mathbf{s}$ and $\mathbf{n}$ align initially $\left(\epsilon_{0} \sim 0\right), i_{0}>90^{\circ}$ when $\gamma_{0}>90^{\circ}$. When the satellite tide is dominant, the second terms of r.h.s. of Eqs. (8) and (10) are negligible. Compared with the prograde cases, $x$ and $y$ change sign while $z$ has the same sign. Then evolution of $\Omega$ and $a$ is the same (Eqs. 10 and 11), while the equations for $x$ and $y$, Eqs. (8) and (9), change sign. So, the obliquity evolution is symmetric about $90^{\circ}$. For $\gamma_{0}>90^{\circ}, \gamma$ decreases toward $90^{\circ}$ until $\Omega z / n<2$. After that the obliquity increases to $180^{\circ}$. In type A3 evolution, however, the secular change in $x$ is dominated by the second term in r.h.s. of Eq. (8) . Then, the obliquity eventually decreases toward $0^{\circ}$.

\subsubsection{Parameter dependence}

Figure 9 shows all the results obtained by numerical calculations. Triangles represent A2 evolution. Type A1 evolution with $\gamma \rightarrow 0^{\circ}$ (for $\gamma_{0}<90^{\circ}$ ) or $\gamma \rightarrow 180^{\circ}$ (for $\gamma_{0}>90^{\circ}$ ) are plotted by open circles. The results with $\gamma \rightarrow 0^{\circ}$ for $\gamma_{0}>90^{\circ}$ (type A3), are plotted by filled squares. For $\gamma_{0}<90^{\circ}$, the boundary between $\mathrm{A} 1$ and $\mathrm{A} 3$ is not clear in the numerical results, so that A3 for $\gamma_{0}<90^{\circ}$ is also plotted by open circles. Type B and C evolution is expressed by crosses and filled circles, respectively. The regions of the qualitatively different tidal evolution are clearly divided in the $m / M_{\mathrm{p}}-\gamma_{0}$ plane. In section 3.2 .2 , we analytically derive the boundaries of the regions.

[Figure 9]

\subsection{Angular momentum and energy}

During the tidal evolution of the system, angular momentum $(L)$ is transfered among the planet, the satellite and the host star, while the total mechanical energy $(E)$ decreases through tidal dissipation in the planet. In this subsection, we first summarize the arguments in terms of $L$ and $E$ for the planar problem developed by Counselman (1972), and generalize it to non-planar systems to explain diversity of tidal evolution found by our numerical simulation. 


\subsubsection{Tidal evolution of co-planar planet-satellite system}

If the stellar torques are not included and $\epsilon_{0}=0$, the tidal evolution is a completely planar problem. Because the stellar tides are neglected, type II and III precessions do not exist. Due to $\epsilon=0$, type I precession does not exist, either. In the planar case, total angular momentum $L$ and total mechanical energy $E$ of the planet-satellite system are given by

$$
\begin{aligned}
& L=H+h=\alpha M_{\mathrm{p}} R_{\mathrm{p}}^{2} \Omega+\frac{M_{\mathrm{p}} m}{M_{\mathrm{p}}+m} n a^{2}, \\
& E=\frac{1}{2} \alpha M_{\mathrm{p}} R_{\mathrm{p}}^{2} \Omega^{2}-\frac{G M_{\mathrm{p}} m}{2 a} .
\end{aligned}
$$

These equations are deduced to non-dimensional forms,

$$
\begin{aligned}
\tilde{L} & =\tilde{\Omega}+\tilde{n}^{-1}, \\
\tilde{E} & =\tilde{\Omega}^{2}-\tilde{n}^{2},
\end{aligned}
$$

where

$$
\begin{aligned}
\tilde{\Omega} & =(\Omega / \sigma) k^{-3} \\
\tilde{n} & =(n / \sigma)^{1 / 3} k^{-1} \\
\tilde{L} & =L\left(\alpha M_{\mathrm{p}} R_{\mathrm{p}}^{2} k^{3} \sigma\right)^{-1} \\
\tilde{E} & =E\left(\alpha M_{\mathrm{p}} R_{\mathrm{p}}^{2} k^{6} \sigma^{2}\right)^{-1} .
\end{aligned}
$$

In the above, the frequency $\sigma$ and a parameter $k$ are defined by $\sigma=\left(G M_{\mathrm{p}} / R_{\mathrm{p}}^{3}\right)^{1 / 2}=(4 \pi G \rho / 3)^{1 / 2}$ and $k=\left(m / \alpha M_{\mathrm{p}}\right)^{1 / 4}\left(1+m / M_{\mathrm{p}}\right)^{-1 / 12}$. The condition of spin-orbit synchronism $(n=\Omega)$ is deduced to

$$
\tilde{\Omega}=\tilde{n}^{3} .
$$

At synchronous points the gradient of $\tilde{E}$ is normal to a constant- $\tilde{L}$ contour.

Contours of $\tilde{L}$ and $\tilde{E}$ and the line $\tilde{\Omega}=\tilde{n}^{3}$ are plotted by solid, dashed, and dotted curves in the $(\tilde{\Omega}, \tilde{n})$ plane in Fig. 10. The satellite-planet system evolves along a constant- $\tilde{L}$ line (corresponding to an initial value, $\tilde{L}_{0}$ ) in the direction of decreasing $\tilde{E}$, as indicated by arrows on solid curves. Note that smaller $|\tilde{n}|$ corresponds to larger $a$. Evolution with decreasing (increasing) $|\tilde{n}|$ represents orbital expansion (decay) of the satellite.

[Figure 10]

The lines of constant- $\tilde{L}$, constant- $\tilde{E}$, and $\tilde{\Omega}=\tilde{n}^{3}$ are mutually tangent at $\left(\tilde{\Omega}^{*}, \tilde{n}^{*}\right)$ and $\left(-\tilde{\Omega}^{*},-\tilde{n}^{*}\right)$, where $\tilde{\Omega}^{*} \simeq 0.439$ and $\tilde{n}^{*} \simeq 0.760$. If $|\tilde{L}|$ is smaller than the critical value $\tilde{L}^{*}=$ $\tilde{L}\left(\tilde{\Omega}^{*}, \tilde{n}^{*}\right)=-\tilde{L}\left(-\tilde{\Omega}^{*},-\tilde{n}^{*}\right) \simeq 1.755$, constant- $\tilde{L}$ line and the synchronism line (Eq. 20) never cross each other, which means that the satellite monotonically migrates inward and inevitably falls onto the planet. If $|\tilde{L}|>\tilde{L}^{*}$, there are two synchronous points for each $\tilde{L}$ : the outer points $\left(a=a_{\mathrm{c}, \text { out }}\right)$ for $|\tilde{\Omega}|<\tilde{\Omega}^{*}$ are stable and the inner ones $\left(a=a_{\mathrm{c}, \text { in }}\right)$ for $|\tilde{\Omega}|>\tilde{\Omega}^{*}$ are unstable, as shown in Fig. 10. 


\subsubsection{Tidal evolution of non-planar planet-satellite system}

In the non-planar cases $(\epsilon \neq 0)$, the total angular momentum is given by

$$
\mathbf{L}=\alpha M_{\mathrm{p}} \Omega R_{\mathrm{p}}^{2} \mathbf{s}+\frac{M_{\mathrm{p}} m}{M_{\mathrm{p}}+m} n a^{2} \mathbf{n} .
$$

Since the stellar torque is considered here, $\mathbf{L}$ changes through exchange with orbital motion of the planet. However, the stellar torque is restricted in the planet's orbital plane (XY plane), $L_{Z}=\mathbf{L} \cdot \mathbf{k}$ is conserved, where

$$
L_{Z}=\alpha M_{\mathrm{p}} \Omega R_{\mathrm{p}}^{2} \cos \gamma+\frac{M_{\mathrm{p}} m}{M_{\mathrm{p}}+m} n a^{2} \cos i .
$$

Note that $L_{Z} \simeq L_{0} \cos \gamma_{0}$, since $\gamma \sim i$ initially.

Figures 11 show the time evolution of normalized $\tilde{L}=|\tilde{\mathbf{L}}|, \tilde{L}_{Z}$, and $\tilde{E}$ (Eqs. 18 and 19), in the cases of (a) $m=0.02 M_{\mathrm{p}}$ and $\gamma_{0}=10^{\circ}$, (b) $m=0.01 M_{\mathrm{p}}$ and $\gamma_{0}=80^{\circ}$ and (c) $m=0.04 M_{\mathrm{p}}$ and $\gamma_{0}=40^{\circ}$. During the evolution, $\tilde{E}$ monotonically decreases throughout the evolution. On the other hand, $\tilde{L}_{Z}$ is conserved in all cases, while $\tilde{L}_{X Y}$ oscillates and secularly decreases because of the stellar torque. Therefore $\tilde{L}$ asymptotically approaches $\tilde{L}_{Z}\left(\simeq \tilde{L}_{0} \cos \gamma_{0}\right)$. Using this characteristic, type B evolution is explicitly predicted by initial parameters $m / M_{\mathrm{p}}$ and $\gamma_{0}$, as below.

\section{[Figure 11]}

In Fig. 12, we plot the numerically obtained time evolution of normalized $\tilde{\Omega} \cos \epsilon$ and $\tilde{n}$ (Eqs. [16 and 17), in the cases of (a) $m=0.02 M_{\mathrm{p}}$ and $\gamma_{0}=10^{\circ}$ (type A evolution in section 3.1.1), (b) $m=0.01 M_{\mathrm{p}}$ and $\gamma_{0}=80^{\circ}$ (type $\mathrm{B}$ in section 3.1.2) and (c) $m=0.04 M_{\mathrm{p}}$ and $\gamma_{0}=40^{\circ}$ (type $\mathrm{C}$ in section 3.1.3). In these figures, we plot $\tilde{\Omega} \cos \epsilon$ instead of $\tilde{\Omega}$ in order to fix the line representing spin-orbit synchronism in the non-coplanar case, $\tilde{\Omega} \cos \epsilon=\tilde{n}^{3}$, on the plane. Contours of $\tilde{L}$ are plotted for $\epsilon=0$. Since $\epsilon$ is not zero generally, actual $\tilde{L}$ is different from $\tilde{L}$ at instantaneous points of trajectories in the figure except for the initial and final stages in which $\epsilon \sim 0$. However, these contours provide good guide for the evolution of the trajectories, as shown below. With $\epsilon_{0} \sim 0$, initial positions are in upper right region $(\tilde{n}>0, \tilde{\Omega}>0)$ or lower left region $(\tilde{n}<0, \tilde{\Omega}<0)$ in Fig. 10. Since evolution is symmetric between the two regions unless stellar tidal torque is dominant, we discuss the cases starting with $\tilde{n}>0$ and $\tilde{\Omega}>0$ below.

In the early phase where type I precession is dominated, $\epsilon \sim 0$ and $\gamma$ and $i$ are conserved. As a consequence, in this phase, the conservation of $\tilde{L}_{Z}$ implies that of $\tilde{L}$ and a trajectory gradually moves along a constant- $\tilde{L}$ line corresponding to $\tilde{L}_{0}$. When the satellite approaches $a_{\text {crit }}$, stellar precession torque becomes important, then $\epsilon$ begins to oscillates on precession periods (type II precession) and $\tilde{L}$ decreases. Because the horizontal axis is $\tilde{\Omega} \cos \epsilon$ in Fig. 12, the oscillation is apparent in the figure. When $\gamma_{0}$ is small, the satellite keeps receding from the planet and approaches $a_{\mathrm{c}, \text { out }}$ (type A evolution in Sec. 3.1), as shown in Fig. 12a. However, in the high $\gamma_{0}$ case (Fig. 12b), the oscillation amplitude of $\epsilon$ is so large that the trajectory strides over $a_{\mathrm{c}, \text { out }}$. The oscillation timescale is comparable to the precession periods of $\mathbf{s}$. Because it may be much shorter than tidal evolution timescale, the system cannot be captured at the synchronism. Once the trajectory strides $a_{\mathrm{c}, \text { out }}$, orbital migration of the satellite is reversed to inward one. In the $\tilde{\Omega} \cos \epsilon-\tilde{n}$ plane in Figs. [12, leftward/downward evolution is reversed to rightward/upward one (see arrows in Fig. 10). 
Due to damping of $\tilde{L}_{X Y}, \tilde{L}$ decreases to $\tilde{L}_{Z}$ after the large oscillation. If $\tilde{L}_{Z}<\tilde{L}_{*} \sim 1.755, \tilde{L}$ becomes smaller than $\tilde{L}_{*}$, so that the satellite eventually falls onto the planet (type B evolution in Sec. 3.1), as shown in Fig. 12b. If $\gamma_{0}$ and hence the oscillation amplitude are not large enough (equivalently, $\tilde{L}_{Z} \simeq \tilde{L}_{0} \cos \gamma_{0}$ is not small enough) to enter the regions of $\tilde{L}<\tilde{L}_{*}$, the satellite meets $a_{\mathrm{c}, \text { out }}$ again during the inward migration (type C evolution in Sec. 3.1), as shown in Fig. 12k. Since the oscillation of $\epsilon$ has been damped, the system is captured at the synchronism. Thus, whether type $\mathrm{B}$ or $\mathrm{C}$ evolution is predicted by initial $\tilde{L}_{Z}$ : type $\mathrm{B}$ for $\tilde{L}_{Z}<\tilde{L}_{*}$ and $\mathrm{C}$ for $\tilde{L}_{Z}>\tilde{L}_{*}$.

\section{[Figure 12]}

\subsection{Co-rotation radius}

The condition to stride $a_{\mathrm{c}, \text { out }}$ separates type $\mathrm{B}$ and $\mathrm{C}$ evolution from type $\mathrm{A}$ evolution. This condition may be roughly obtained by $a_{\text {crit }} \gtrsim a_{\mathrm{c}, \text { out }}^{\infty}$, where $a_{\mathrm{c}, \text { out }}^{\infty}$ is the outer co-rotation radius evaluated with $\gamma=i=\epsilon=0$. As shown in next subsection, this condition is in good agreement with the numerical results.

The expression of $a_{\mathrm{c}, \text { out }}^{\infty}$ is derived by the conservation of $L_{Z} . L_{Z}$ at $a_{0}$ is

$$
\begin{aligned}
L_{Z 0} & \simeq\left(\alpha M_{\mathrm{p}} \Omega_{0} R_{\mathrm{p}}^{2}+\frac{M_{\mathrm{p}} m}{M_{\mathrm{p}}+m} n\left(a_{0}\right) a_{0}^{2}\right) \cos \gamma_{0} \equiv \alpha M_{\mathrm{p}} \Omega_{0} R_{\mathrm{p}}^{2}\left(1+f_{0}\right) \\
f_{0} & \simeq 0.2\left(\frac{\alpha}{0.33}\right)^{-1}\left(\frac{\rho}{5.5 \mathrm{gcm}^{-3}}\right)^{1 / 2}\left(\frac{m}{0.01 M_{\mathrm{p}}}\right)\left(\frac{D_{0}}{5 \mathrm{hrs}}\right)\left(\frac{a_{0}}{3.8 R_{\mathrm{p}}}\right)^{1 / 2},
\end{aligned}
$$

where subscript " 0 " represents initial values. Using the definition of the co-rotation radius $\left(a_{\mathrm{c}}\right)$, $\Omega\left(a_{\mathrm{c}}\right) \cos \epsilon=n\left(a_{\mathrm{c}}\right)$, the angular momentum at $a_{\mathrm{c}}$ is

$$
L_{Z \mathrm{c}}=\alpha M_{\mathrm{p}} n\left(a_{\mathrm{c}}\right) R_{\mathrm{p}}^{2} \frac{\cos \gamma}{\cos \epsilon}+\frac{M_{\mathrm{p}} m}{M_{\mathrm{p}}+m} n\left(a_{\mathrm{c}}\right) a_{\mathrm{c}}{ }^{2} \cos i .
$$

Assuming that at $a_{\mathrm{c}, \text { out }}, i \simeq 0$ and the satellite orbital angular momentum is dominated, $L_{Z \mathrm{c}} \simeq m n\left(a_{\mathrm{c}, \text { out }}\right) a_{\mathrm{c}, \text { out }}{ }^{2} \simeq m \sqrt{G M_{\mathrm{p}} a_{\mathrm{c}, \text { out }}}$. From $L_{Z 0}=L_{Z \mathrm{c}}$,

$$
\begin{aligned}
\frac{a_{\mathrm{c}, \text { out }}^{\infty}}{R_{\mathrm{p}}} & \sim \alpha^{2}\left(\frac{\Omega_{0}}{\sigma}\right)^{2}\left(1+f_{0}\right)^{2}\left(\frac{m}{M_{\mathrm{p}}}\right)^{-2} \cos ^{2} \gamma_{0} \\
& \left.\simeq 89\left(\frac{\alpha}{0.33}\right)^{2}\left(\frac{\rho}{5.5 \mathrm{gcm}^{-3}}\right)^{-1 / 3}\left(1+f_{0}\right)^{2}\left(\frac{m}{0.01 M_{\mathrm{p}}}\right)^{-2}\left(\frac{D_{0}}{5 \mathrm{hrs}}\right)^{-2}\left(\frac{M_{\mathrm{p}}}{M_{\oplus}}\right)^{-2 / 3} \cos ^{2}(27)\right)
\end{aligned}
$$

Substitution of $m=0.02 M_{\mathrm{p}}$ and $\gamma_{0}=10^{\circ}$ yields $a_{\mathrm{c}, \text { out }} \sim 42 R_{\mathrm{p}}$, which is consistent with the numerical results in Fig. [5d.

\subsection{Classification of tidal evolution}

[Figure 13]

Summarizing the above analytical arguments on the tidal evolution, the $m / M_{\mathrm{p}^{-}} \gamma_{0}$ plane is partitioned into three regions labeled A, B and C as shown in Fig. 13. In region A, the satellite

monotonically recedes from the planet until $a$ reaches $a_{\mathrm{c}, \text { out }}$. With parameters in region A1, 
$a_{\mathrm{c}, \text { out }}^{\infty}$ is larger than $a_{\text {crit }}$, so that synchronous state $\Omega=n$ with $\gamma \sim i \sim \epsilon \sim 0$ is realized. With parameters in region A2, synchronous state is achieved before $a$ reaches $\sim a_{\text {crit. }}$ Then, the obliquity and inclinations $(\gamma, i$ and $\epsilon)$ do not vary from initial values. In this case, since $\gamma_{0} \sim \gamma \sim i, a_{\mathrm{c} \text {,out }}$ is given by Eq. (27) without the factor $\cos ^{2} \gamma_{0}$. Hence the boundary is independent of $\gamma_{0}$. In region $A 3$, because of small satellite mass, the obliquity evolution is dominated by the stellar tidal torque. In regions $\mathrm{B}$ and $\mathrm{C}$, the satellite's orbit expands until $a_{\text {crit }}$. At $a_{\text {crit }}, \epsilon$ begins to oscillate by the character change of precessional motion so that the satellites begin to migrate inward. The satellite stops at $a_{\mathrm{c}, \text { out }}$ for the parameters in region $\mathrm{C}$, while it falls onto the planet in region $\mathrm{B}$.

Regions $\mathrm{B}$ and $\mathrm{C}$ are separated by $\tilde{L}_{\mathrm{Z} 0}=1.755$, which is represented by the dashed line in Fig. 13. The boundary of $\mathrm{A} 1$ from $\mathrm{B}$ or $\mathrm{C}$ is given by $a_{\mathrm{crit}}=a_{\mathrm{c}, \text { out }}^{\infty}$, represented by the solid line in Fig. 13. If $m$ exceeds the mass determined by the above equation with $\gamma_{0}=0^{\circ}, a_{\mathrm{c}, \text { out }}^{\infty}$ is smaller than $a_{\text {crit }}$ for any $\gamma_{0}$. This separates A2 from B or C. The stellar tidal effects are stronger than satellite's one if the timescale $\left(\Delta t_{\mathrm{A} 1}\right)$ for orbital expansion to $a_{\mathrm{c}, \text { out }}^{\infty}$ is longer than the timescale $\left(\Delta t_{\mathrm{A} 3}\right)$ required for the synchronism by the stellar tide $\left(\Delta t_{\mathrm{A} 1}\right.$ and $\Delta t_{\mathrm{A} 3}$ are given in section 3.5). This separates A3 from A1.

In Fig. 14, we compare these analytical boundaries with the numerical results in Fig. 9. Circles and filled squares represent A1 and A3 evolution, respectively. In the numerical calculation, A3 evolution with $\gamma_{0}<90^{\circ}$ is similar to A1 evolution. Triangles, crosses and filled circles represents $\mathrm{A} 2, \mathrm{~B}$ and $\mathrm{C}$ evolution, respectively. The analytically estimated boundaries are in good agreement with the numerical results, especially the boundary of A1 from B or C and that separating B and C. Since the transition of precession from type I to II is not determined exactly by Eq. (7), the boundary which separates $\mathrm{A} 2$ from $\mathrm{B}$ or $\mathrm{C}$ is not clear enough in the numerical calculation.

[Figure 14]

\subsection{Variation magnitude and timescales of obliquity evolution}

So far we have been concerned with diversity of tidal evolution and intrinsic dynamics that regulates the diversity. In this subsection, we discuss ranges of the obliquity changes and their timescales that may be important for implications for planetary climate. Typical obliquity changes in individual types of evolution are illustrated in Fig. 15. In general, the variation $\Delta \gamma$ in the obliquity is large for large $\sin \gamma_{0}$. For $\mathrm{B}$ and $\mathrm{C}$ evolution, not only the precessionaveraged $\Delta \gamma$ but also the oscillation amplitude of $\gamma$ during precession is large after the satellite's migration turns back.

\section{[Figure 15]}

The evolution timescales of the obliquity in A1 and A2 are comparable to migration timescale to $a_{\mathrm{c} \text {,out }}$. The migration timescale depends on the specific dissipation function $Q$, which is defined as the inverse of the frictional energy dissipation per cycle of the tidal oscillation, related to the phase shift $\delta$ as $1 / Q \sim 2 \delta \sim 2 \delta t(\Omega-n)$ (e.g., Murray and Dermott 1999). With $Q$, Eq. (11) is approximately written as

$$
\frac{d a}{d t}=\operatorname{sign}(\Omega-n) \frac{3 k_{2}}{Q} \frac{m}{M_{\mathrm{p}}}\left(\frac{R_{\mathrm{p}}}{a}\right)^{5} n a .
$$


Integrating this equation from $a_{0}$ to $a_{\mathrm{c}, \text { out }}$ is

$$
\Delta t=\frac{2}{13}\left(\frac{1}{12 \pi G}\right)^{1 / 2} \frac{Q}{k_{2} \rho^{1 / 2}}\left(\frac{M_{\mathrm{p}}}{m}\right)\left\{\left(\frac{a_{\mathrm{c}}}{R_{\mathrm{p}}}\right)^{13 / 2}-\left(\frac{a_{0}}{R_{\mathrm{p}}}\right)^{13 / 2}\right\} .
$$

(The term of $a_{0}$ is negligible.) Substituting Eq. (27) into Eq. (29), the timescale for A1 evolution is

$$
\begin{aligned}
\Delta t_{\mathrm{A} 1} \sim 2 \times 10^{10}\left(\frac{Q}{10}\right) & \left(\frac{k_{2}}{0.3}\right)^{-1}\left(\frac{\alpha}{0.33}\right)^{13}\left(\frac{\rho}{5.5 \mathrm{gcm}^{-3}}\right)^{-7} \\
& \times\left(1+f_{0}\right)^{13}\left(\frac{m}{0.01 M_{\mathrm{p}}}\right)^{-14} \cos ^{13} \gamma_{0} \quad \text { [year] }
\end{aligned}
$$

Timescale for A2 evolution is given by Eq. (30) with $\gamma_{0}=0^{\circ}$ for any $\gamma_{0}$. Figure 16 shows A1 evolution time calculated by Eq. (29) with $Q=12$ (current Earth's value) and $k_{2}=0.30$. Note that $\Delta t_{\mathrm{A} 1}$ strongly depends on the mass ratio $m / M_{\mathrm{p}}$ and the initial obliquity $\gamma_{0}$. Also note that $Q$ could be larger than the current Earth's value for planets in early stage, so that the estimate of evolution timescale includes some uncertainty.

\section{[Figure 16]}

The satellite's orbital evolution of type $\mathrm{B}$ and $\mathrm{C}$ is expansion to $a_{\text {crit }}$ followed by inward migration to $a_{\mathrm{c}, \text { out }}$ or to the planetary surface. The characteristic evolution timescales are given by integrating Eq. (28) to $a_{\text {crit }}$ and multiplying it by a factor 2. Replacing $a_{\text {c }}$ by $a_{\text {crit }}$ in Eq. (29) and $\times 2$, and substituting Eq. (7) into it,

$$
\begin{aligned}
\Delta t_{\mathrm{B}, \mathrm{C}} \sim 1.5 \times 10^{6} & \left(\frac{Q}{10}\right)\left(\frac{k_{2}}{0.3}\right)^{-1}\left(\frac{M_{*}}{1 M_{\odot}}\right)^{-13 / 10}\left(\frac{a_{\mathrm{p}}}{1 \mathrm{AU}}\right)^{39 / 10} \\
& \times k_{s}^{13 / 10}\left(\frac{\rho}{5.5 \mathrm{gcm}^{-3}}\right)^{-1 / 2}\left(\frac{D}{5 \mathrm{hrs}}\right)^{-13 / 5}\left(\frac{m}{0.01 M_{\mathrm{p}}}\right)^{-1}[\text { year }] .
\end{aligned}
$$

The A3 evolution timescale is the time necessary for $\Omega$ to be decreased to orbital mean motion $n_{\mathrm{p}}$ by the stellar torque. Neglecting the first term in r.h.s. of Eq. (10),

$$
\frac{d \Omega}{d t} \simeq-\frac{3 k_{2}}{2 \alpha Q}\left(\frac{M_{*}}{M_{\mathrm{p}}}\right)\left(\frac{R_{\mathrm{p}}}{a_{\mathrm{p}}}\right)^{3} n_{\mathrm{p}}^{2}
$$

Integrating Eq. (32) from $\Omega_{0}$ to $n_{\mathrm{p}}=\left(G M_{*} / a_{\mathrm{p}}^{3}\right)^{1 / 2}$,

$$
\begin{aligned}
\Delta t_{\mathrm{A} 3}= & \frac{4 \pi}{3}\left(\frac{Q}{k_{2}}\right) \frac{\alpha \rho}{G} \frac{a_{\mathrm{p}}^{6}}{M_{*}^{2}}\left(\Omega_{0}-n_{\mathrm{p}}\right) \\
\sim & 3 \times 10^{11}\left(\frac{Q}{10}\right)\left(\frac{k_{2}}{0.3}\right)^{-1}\left(\frac{M_{*}}{1 M_{\odot}}\right)^{-2}\left(\frac{a_{\mathrm{p}}}{1 \mathrm{AU}}\right)^{6} \\
& \times\left(\frac{\alpha}{0.33}\right)\left(\frac{\rho}{5.5 \mathrm{gcm}^{-3}}\right)\left(\frac{D_{0}}{5 \mathrm{hrs}^{-1}}\right. \text { [year] }
\end{aligned}
$$




\section{Discussion}

Here we comment on the spin-orbit resonances along the tidal evolution. Other planets' gravitational perturbations cause precession of the planetary orbit about the invariant plane of the planetary system. When the spin axis precession frequency $\left(\nu_{\mathrm{s}}\right)$ is commensurate with one of eigenvalues of the orbital precession frequency $\left(\nu_{\mathrm{o}}\right)$, the spin axis can fluctuate with large amplitudes. Ward (1973) pointed out that Mars' obliquity has suffered from a large scale oscillation because of these spin-orbit resonances. Laskar and Robutel (1993) showed that in the absence of massive satellites all of the terrestrial planets could have experienced large-scale obliquity variations. Atobe et al. (2004) showed that terrestrial planets in habitable zones in extrasolar planetary systems with a gas giant(s) generally tend to undergo the spin-orbit resonance if they do not have satellites. They assumed that the planets without satellites underwent nearly head-on collisions and had relatively slow spin ( $D \gtrsim 20$ hours). Due to the relatively large mass of the Moon, Earth's spin axis precesses rapidly and avoids the orbital precession frequencies of the planets (Ward 1974; Laskar and Robutel 1993).

As the orbit expands due to the tidal evolution, satellite's contribution to the spin axis precession gradually decreases. Initially, $\nu_{\mathrm{s}} \gg \nu_{\mathrm{o}} \sim 10^{-4}-10^{-5} \mathrm{rad} /$ year, because of small $D$ and $a$. Because a satellite was formed, the impact must have been grazing one, so that initial $D$ may be $\sim 5$ hours or less. Since $D$ and $a$ increase through the tidal evolution, $\nu_{\mathrm{s}}$ decreases. If a satellite is more massive than the lunar mass $\left(\sim 0.01 M_{\oplus}\right)$, the expansion of $a$ is limited because of small $a_{\mathrm{c}, \text { out }}$ (Eq. 27). Then the decrease of $\nu_{\mathrm{s}}$ stops before it goes down to the level of $\nu_{\mathrm{o}}$, so that the spin-orbit resonance is avoided. If a satellite is light enough, the expansion is so slow that spin-orbit resonance is not realized during main-sequence lifetime $\left(\sim 10^{10}\right.$ years $)$ of solar-type stars. If a satellite has comparable mass to the lunar mass, $\nu_{\mathrm{s}}$ eventually becomes as small as $\nu_{\mathrm{o}}$ within $10^{10}$ years through tidal evolution, and then obliquity may suffer large variation by the spin-orbit resonance. Hence, the spin-orbit resonance may be important for planets without satellites (always) and for planets with satellites of about the lunar mass (at some point in tidal evolution within main-sequence lifetime).

So far, we have been concerned with planets at $\sim 1 \mathrm{AU}$, that is, planets in habitable zones around solar-type stars $\left(M_{*} \sim 1 M_{\odot}\right)$. Habitable zones around lower-mass stars (e.g., M dwarfs) are well inside 1AU, because of the low stellar luminosity. As shown in Eq. (34), small $a_{\mathrm{p}}$ leads to rapid removal of angular momentum from the planet-satellite system. Whatever divergent tidal evolution the system has undergone, the satellite eventually falls to the planet and the planet spin eventually becomes straight and synchronous with its orbital motion by the stellar tidal effects within $10^{8}$ years. Thus, planets in habitable zones have diversity in planet-satellite configurations during main-sequence phase of host stars, if the stars are solar-type stars or more massive stars.

Final accretion stage of terrestrial planets would be multiple collisions of protoplanets (see Sec. 1). The probability of a grazing impact to produce a satellite may be higher than head-on one. Since the spin produced by the impact is more likely to be tilted, the formed satellite may be perished onto the planetary surface through type B tidal evolution on timescales of Myrs or trapped near the planetary surface through type $\mathrm{C}$ evolution. This means that the satellite may orbit near the planetary surface when a next protoplanet approaches the planet. Velocity dispersion of protoplanets would be similar to their surface escape velocity, which is also similar to Keplerian velocity of the satellite in the proximity of the planetary surface. Hence, the energy and angular momentum exchange with the satellite could alter the orbit of the approaching protoplanet. This effect might alter scattering/collision cross sections of protoplanets. 


\section{Conclusion}

We have investigated obliquity evolution of terrestrial planets due to tidal interaction with their satellites and host stars by numerical integration and analytical arguments, with wide variety of initial conditions on the basis of recent N-body simulations of planet accretion. We found three domains in the parameters of satellite-to-planet mass ratio $\left(m / M_{\mathrm{p}}\right)$ and initial obliquity $\left(\gamma_{0}\right)$ in which the evolution is qualitatively different from one another.

If a satellite is formed from a debris disk created by an off-center giant impact and the planetary spin is dominated by the impact, the planet would have various initial obliquity and a satellite with various mass. And planetary spin axis and orbit normal of the satellite are almost aligned $(\epsilon \sim 0)$. Since N-body simulations show that the impacts are almost isotropic, an initial planetary spin axis is more likely to be tilted $\left(\sin \gamma_{0} \sim 1\right)$ from the planet orbit normal than to be aligned with it $\left(\sin \gamma_{0} \sim 0\right)$. In order to study tidal evolution of extrasolar terrestrial planet- satellite systems, we numerically integrated evolution with various $m / M_{\mathrm{p}}$ and various initial $\gamma_{0}$, focusing on tilted ones. (We consider the regions at $\sim 1 \mathrm{AU}$ and assume $\epsilon_{0} \sim 0$.) Most of previous studies on tidal evolution, however, have focused on a particular case of the Earth-Moon systems in which $m / M_{\mathrm{p}}=0.0125$ and $\gamma_{0} \sim 10^{\circ}$ (e.g., Goldreich 1966; Mignard 1978, 1979, 1980; Touma and Wisdom 1994) or the 2-body planar problem $\gamma=0^{\circ}$ (e.g., Counselman 1979).

As the previous studies show, the satellite orbit first expands with the almost constant obliquity $(\gamma)$, the inclination of the satellite orbit normal to the planet one $(i)$, and that to the planetary spin axis $(\epsilon)$ until orbital radius of the satellite $(a)$ reaches $\sim a_{\text {crit }} \sim 15 R_{\mathrm{p}}$ (Eq. 7 ) at which the precession about total angular momentum vector is transformed into that about the planet orbital normal. We have found that this character change in precession causes diversity of tidal evolution as follows:

1. At $a \sim a_{\text {crit }}, \epsilon$ starts oscillation between $\sim 0$ and $\sim 2 \gamma_{0}$. The enhanced $\epsilon$ instantaneously reduces outer co-rotation radius $\left(a_{\mathrm{c}, \text { out }}\right)$. For large $\sin \gamma_{0}$ cases, $a_{\mathrm{c}, \text { out }}$ strides across $a$ without capture at the synchronism and the outward migration of the satellite orbit is reversed to inward one.

2. The evolution with the migration reversal is further divided into two types. The initial $L_{Z}$ determines whether the turned-back satellite falls onto the planet (type B evolution) or it is captured at $a_{\mathrm{c} \text {,out }}$ that has jumped inward to $\sim 5-10 R_{\mathrm{p}}$ (type $\mathrm{C}$ evolution).

3. With the analytical arguments, the parameter space of $m / M_{\mathrm{p}}$ and $\gamma_{0}$ is divided into three domains (A, B, and C) of qualitatively different tidal evolution (Fig. 13), which is in good agreement with the numerical integration (Fig. 14). Type A evolution is further divided into three types: A1) evolution similar to the Earth-Moon system, A2) evolution with the synchronism at $<a_{\text {crit }}$ for $m / M_{\mathrm{p}} \gtrsim 0.05$, and A3) evolution dominated by stellar tidal torque for $m / M_{\mathrm{p}} \lesssim 0.005$.

4. In the final state approaching $a_{\mathrm{c} \text {,out }}$ or the planetary surface, $\epsilon \sim 0^{\circ}$. On the other hand, $\gamma, i \sim 0^{\circ}$ for $\gamma_{0}<90^{\circ}$ while $\gamma, i \sim 180^{\circ}$ for $\gamma_{0}>90^{\circ}$ except for A3 evolution. Typical $\gamma$ evolution in each type is shown in Fig. 15. The variation timescales are evaluated in Figs. 16. The timescales for $\mathrm{B}$ and $\mathrm{C}$ evolution are relatively short $\left(\lesssim 10^{6}\right.$ years $)$, because the satellite orbit turns back at relatively small radius $a_{\text {crit }}$.

If the formation of a satellite that is comparable to or larger than the Moon's mass and planetary spin state are determined by a giant impact, the tidal evolution of the satellite orbit 
and the spin is most likely to be type $\mathrm{B} / \mathrm{C}$ that have been discovered by the present paper. (We will elsewhere discuss the predicted distributions of $m / M_{\mathrm{p}}$ and $\gamma_{0}$.) To address dynamics of terrestrial planet-satellite systems in extrasolar planetary systems, it is important to consider the large diversity of tidal evolution. This diversity may be also important to discuss the evolution of surface environments of extrasolar terrestrial planets and their habitability.

\section{Acknowledgments}

This work was supported by grant-in-aid, MEXT 16077202. We thank anonymous referees for helpful comments to improve the paper. 


\section{Appendix A: Equations of precessional motions}

Here we briefly summarize the precessional torques and detailed equations of precessional motions derived by Goldreich (1966). The torques acting on the planet $\left(\mathbf{L}_{\mathrm{p}}\right)$ is sum of those from the satellite and the star: $\mathbf{L}_{\mathrm{p}}=\mathbf{L}_{\mathrm{ps}}+\mathbf{L}_{\mathrm{p} *}$. Similarly, torques acting on the satellite is $\mathbf{L}_{\mathrm{s}}=\mathbf{L}_{\mathrm{sp}}+\mathbf{L}_{\mathrm{s} *}$, where subscripts "s", "p" and "*" represent the satellite, the planet and the star respectively. $\mathbf{L}_{\mathrm{sp}}$ averaged over the orbital periods is given by

$$
\mathbf{L}_{\mathrm{sp}}=-\frac{G M_{\mathrm{p}} m}{a^{3}} J_{2} R_{\mathrm{p}}^{2}(\mathbf{s} \cdot \mathbf{n})(\mathbf{s} \times \mathbf{n}) \equiv-L(\mathbf{s} \cdot \mathbf{n})(\mathbf{s} \times \mathbf{n}),
$$

where $a$ is the semi-major axis of the satellite's orbit, $\sigma=\left(G M_{\mathrm{p}} / R_{\mathrm{p}}^{3}\right)^{1 / 2}$, and $J_{2}$ is the planetary oblateness coefficient given by

$$
J_{2}=\frac{k_{\mathrm{s}} R_{\mathrm{p}}^{3} \Omega^{2}}{2 G M_{\mathrm{p}}}=\frac{k_{\mathrm{s}}}{2} \frac{\Omega^{2}}{\sigma^{2}},
$$

( $k_{\mathrm{s}}$ is the secular Love number). For the current Earth, $k_{\mathrm{s}}=0.95$ as to give the known value of $J_{2}$ (Munk and MacDonald 1960). Replacing $m$ and $a$ in Eq. (35) by $M_{*}$ and $a_{\mathrm{p}}$, the averaged precessional torque exerted by the planetary equatorial bulge on the host $\operatorname{star}\left(\mathbf{L}_{* \mathrm{p}}\right)$ is

$$
\mathbf{L}_{* \mathrm{p}}=-\frac{G M_{*} M_{\mathrm{p}}}{a_{\mathrm{p}}^{3}} J_{2} R_{\mathrm{p}}^{2}(\mathbf{s} \cdot \mathbf{k})(\mathbf{s} \times \mathbf{k}) \equiv-K_{1}(\mathbf{s} \cdot \mathbf{k})(\mathbf{s} \times \mathbf{k}),
$$

where $a_{\mathrm{p}}$ is the semi-major axis of the planet's orbit. The averaged torque exerted by the satellite on the $\operatorname{star}\left(\mathbf{L}_{* \mathrm{~s}}\right)$ is

$$
\mathbf{L}_{* \mathrm{~s}}=-\frac{3}{4} \frac{G M_{*} m}{a_{\mathrm{p}}^{3}} a^{2}(\mathbf{n} \cdot \mathbf{k})(\mathbf{n} \times \mathbf{k}) \equiv-K_{2}(\mathbf{n} \cdot \mathbf{k})(\mathbf{n} \times \mathbf{k}) .
$$

Since the satellite is regarded as a ring with radius $a$ and mass $m$ in the orbit averaging, $M_{\mathrm{p}} R_{\mathrm{p}}^{2} J_{2}$ in Eq. (37) is replaced by $\sim m a^{2}$ in Eq. (38).

Since $\mathbf{L}_{\mathrm{p} *}=-\mathbf{L}_{* \mathrm{p}}, \mathbf{L}_{\mathrm{ps}}=-\mathbf{L}_{\mathrm{sp}}, \mathbf{L}_{\mathrm{s} *}=-\mathbf{L}_{* \mathrm{~s}}$,

$$
\begin{aligned}
\frac{d H \mathbf{s}}{d t} & =L(\mathbf{s} \cdot \mathbf{n})(\mathbf{s} \times \mathbf{n})+K_{1}(\mathbf{s} \cdot \mathbf{k})(\mathbf{s} \times \mathbf{k}), \\
\frac{d h \mathbf{n}}{d t} & =-L(\mathbf{s} \cdot \mathbf{n})(\mathbf{s} \times \mathbf{n})+K_{2}(\mathbf{n} \cdot \mathbf{k})(\mathbf{n} \times \mathbf{k}) .
\end{aligned}
$$

Taking the dot products of Eqs. (39) and (40) with $\mathbf{s}$ and $\mathbf{n}$, respectively, $d H / d t=d h / d t=0$. These equations yield Eqs. (3) and (4).

Dotting $\mathbf{k}$ into Eqs. (3) and (44) and forming the combination $h H d(\mathbf{s} \cdot \mathbf{n}) / d t$,

$$
\begin{aligned}
\frac{d x}{d t} & =\frac{L}{H} z w \\
\frac{d y}{d t} & =-\frac{L}{h} z w \\
\frac{d z}{d t} & =\left(\frac{K_{2}}{h} y-\frac{K_{1}}{H} x\right) w
\end{aligned}
$$

where $x=\mathbf{s} \cdot \mathbf{k}=\cos \gamma, y=\mathbf{n} \cdot \mathbf{k}=\cos i, z=\mathbf{s} \cdot \mathbf{n}=\cos \epsilon$ and

$$
w^{2}=[(\mathbf{s} \times \mathbf{n}) \cdot \mathbf{k}]^{2}=1-x^{2}-y^{2}-z^{2}+2 x y z .
$$


From Eqs. (41), (42) and (43), we obtain the new constants

$$
\begin{aligned}
\Lambda_{Z} & =H x+h y, \\
\chi & =K_{1} x^{2}+K_{2} y^{2}+L z^{2} .
\end{aligned}
$$

The conservation of $\Lambda_{z}$ arises because the external (stellar) torques on the planet-satellite system lie in the planet's orbit. $\chi$ is a kind of potential energy. Differentiating Eq. (44) with Eq. (41) to (43) yields

$$
\frac{d w}{d t}=\frac{L}{H} z(y z-x)-\frac{L}{h} z(x z-y)+\left(\frac{K_{2}}{h} y-\frac{K_{1}}{H} x\right)(x y-z) .
$$

The numerical methods are as follows. Precessional motions are calculated by determining initial data for $x, y$, and $z$ using Eqs. (45), (46) and (44), and $w$ with $w=0$ without any loss of generality, and simultaneously integrating $x$ (Eq. 41), $z$ (Eq. 43), and $w$ (Eq. 47) with the conservation law for $\Lambda_{Z}$ (Eq. 45) to eliminate $y$, using a 4-th order Runge-Kutta method. Then we average the tidal torques given by Eqs. (55) to (66) in Appendix C over one precessional period with the resulting (instantaneous) values of $x, y, z$, and $w$. Tidal evolution is calculated by integrating the tidal equations given by $d H / d t$ (Eq. 48), $d \Lambda_{Z} / d t$ (Eq. 51), $d a / d t$ (Eq. 54), and $d \chi / d t$ (Eq. 52) with the updated averaged torques and $x, y, z$ in the previous step. With the updated $H, \Lambda_{Z}, a$, and $\chi$, we go back to the calculation of precessional motions.

This method of integration assumes that the three timescales of orbit, precession and tidal evolution are well separated. In some cases, the precessional periods can be comparable to or longer than the tidal evolution timescale. In the cases, we also integrate directly Eqs. (11) and (2), instead of the hierarchical method. We found that the hierarchical method produces the results in good agreement with direct method.

\section{Appendix B. Equations of tidal evolution}

In the planet-satellite-star system, the principal tidal change is brought about by the frictionally retarded tide on the planet by the satellite, which results in a loss of mechanical energy from the planet-satellite system and angular momentum transfer from the planet's spin to the orbital motion of the satellite. Additional smaller change produced by tide due to the host star results in dissipation of energy and angular momentum transfer from the planet-satellite system to orbital motions about the star. Assuming a phase-locked spin of the satellite on a circular orbit, the tide by the planet on the satellite does not lead to any secular change. Thus, we only consider planetary tides induced by the star and the satellite.

In Appendix B and C, all quantities are precession-averaged ones. For simplicity, notations for the averaging are omitted. Dotting Eqs. (5) by $\mathbf{s}$ and $\mathbf{n}$ yields

$$
\frac{d H}{d t}=\mathbf{T}_{\mathrm{p}} \cdot \mathbf{s}, \frac{d h}{d t}=\mathbf{T}_{\mathrm{s}} \cdot \mathbf{n}
$$

Dotting Eqs. (5) by $\mathbf{k}$ yields

$$
\begin{aligned}
& \frac{d x}{d t}=\frac{\mathbf{T}_{\mathrm{p}} \cdot \mathbf{k}-x \mathbf{T}_{\mathrm{p}} \cdot \mathbf{s}}{H} \\
& \frac{d y}{d t}=\frac{\mathbf{T}_{\mathrm{s}} \cdot \mathbf{k}-y \mathbf{T}_{\mathrm{s}} \cdot \mathbf{n}}{h}
\end{aligned}
$$


From these equations,

$$
\frac{d \Lambda_{Z}}{d t}=\left(\mathbf{T}_{\mathrm{p}}+\mathbf{T}_{\mathrm{s}}\right) \cdot \mathbf{k}
$$

Differentiating Eq. (46) yields

$$
\frac{d \chi}{d t}=\frac{2 K_{1}}{H} x \mathbf{T}_{\mathrm{p}} \cdot \mathbf{k}+\frac{2 K_{2}}{h} y\left(\mathbf{T}_{\mathrm{s}} \cdot \mathbf{k}+y \mathbf{T}_{\mathrm{s}} \cdot \mathbf{n}\right) .
$$

From Eq. (48) with $H=I_{\mathrm{p}} \Omega$,

$$
\frac{d \Omega}{d t}=\frac{\mathbf{T}_{\mathrm{p}} \cdot \mathbf{s}}{I_{\mathrm{p}}},
$$

where $I_{\mathrm{p}}$ is the planet's moment of inertia, given by $\alpha M_{\mathrm{p}} R_{\mathrm{p}}^{2}$. Since $h=m n a^{2}$, Eq. (48) yields

$$
\frac{d a}{d t}=2 a \frac{\mathbf{T}_{\mathbf{s}} \cdot \mathbf{n}}{h}
$$

\section{APPENDIX C: The averaged tidal torques}

In the present paper, we only consider frictional dissipation due to tidal deformation of the planet caused by the satellite and the star. The averaged tidal torques on the satellite $\left(\mathbf{T}_{\mathrm{s}}\right)$ is the sum of the torque by the satellite tide $\left(\mathbf{T}_{\mathrm{ss}}\right)$ and that by the stellar tide $\left(\mathbf{T}_{s *}\right)$. If the spin axis is not aligned with the orbit normal of the tide raising body, the spin can carry the tidal bulge out of the orbital plane. This out-of-plane bulge can produce in-plane torques on a third body. Similarly, the torques on the star $\left(\mathbf{T}_{*}\right)$ is the sum of $\mathbf{T}_{* *}$ (due to the stellar tide) and $\mathbf{T}_{* \mathrm{~s}}$ (due to the satellite tide). The torques on the planet are the opposite of the torques on the exterior bodies, $\mathbf{T}_{\mathrm{p}}=-\left(\mathbf{T}_{*}+\mathbf{T}_{s}\right)=-\left(\mathbf{T}_{* *}+\mathbf{T}_{* s}+\mathbf{T}_{\mathrm{s} *}+\mathbf{T}_{\mathrm{ss}}\right)$.

Adopting the constant time lag model by Mignard (1981) and Touma and Wisdom (1994), in which the distortion of the planet is delayed from the tide raising potential by the time lag $\delta t$, the torques are

$$
\begin{aligned}
\mathbf{T}_{\mathrm{ss}} \cdot \mathbf{s} & =\delta t \frac{k_{2} G m^{2} R_{\mathrm{p}}^{5}}{a^{6}}\left[\frac{3}{2} \Omega \sin ^{2} \epsilon+3 \cos \epsilon(\Omega \cos \epsilon-n)\right], \\
\mathbf{T}_{\mathrm{ss}} \cdot \mathbf{n} & =\delta t \frac{k_{2} G m^{2} R_{\mathrm{p}}^{5}}{a^{6}}[3(\Omega \cos \epsilon-n)], \\
\mathbf{T}_{\mathrm{ss}} \cdot \mathbf{k} & =\delta t \frac{k_{2} G m^{2} R_{\mathrm{p}}^{5}}{a^{6}}\left[\frac{3}{2} \Omega(\cos \gamma-\cos i \cos \epsilon)+3 \cos i(\Omega \cos \epsilon-n)\right],
\end{aligned}
$$

where $k_{2}$ is the dimensionless tidal Love number (Munk and MacDonald 1960), and

$$
\begin{aligned}
\mathbf{T}_{* *} \cdot \mathbf{s} & =\delta t \frac{k_{2} G M_{*}^{2} R_{\mathrm{p}}^{5}}{a_{p}^{6}}\left[\frac{3}{2} \Omega \sin ^{2} \gamma+3 \cos \gamma\left(\Omega \cos \gamma-n_{\mathrm{p}}\right)\right], \\
\mathbf{T}_{* *} \cdot \mathbf{n} & =\delta t \frac{k_{2} G M_{*}^{2} R_{\mathrm{p}}^{5}}{a_{p}^{6}}\left[\frac{3}{2} \Omega(\cos \epsilon-\cos \gamma \cos i)+3 \cos i\left(\Omega \cos \gamma-n_{\mathrm{p}}\right)\right], \\
\mathbf{T}_{* *} \cdot \mathbf{k} & =\delta t \frac{k_{2} G M_{*}^{2} R_{\mathrm{p}}^{5}}{a_{p}^{6}}\left[3\left(\Omega \cos \gamma-n_{\mathrm{p}}\right)\right] .
\end{aligned}
$$


Denoting the longitude of the ascending node of the planet's equatorial plane on its orbital plane and that of the satellite's orbital plane on the planet's orbital plane as $\Phi$ and $\Psi$,

$$
\begin{aligned}
\mathbf{T}_{\mathrm{s} *} \cdot \mathbf{s}= & \Omega \delta t \frac{k_{2} G m M_{*} R_{\mathrm{p}}^{5}}{a^{3} a_{p}^{3}}\left[\frac{3}{8} \sin ^{2} i \sin ^{2} \gamma \cos 2(\Phi-\Psi)-\frac{9}{8} \sin ^{2} i \sin ^{2} \gamma\right. \\
& \left.-\frac{3}{4} \cos \gamma \sin \gamma \cos i \sin i \cos (\Phi-\Psi)+\frac{3}{4} \sin ^{2} \gamma\right], \\
\mathbf{T}_{\mathrm{s} *} \cdot \mathbf{n}= & 0 \\
\mathbf{T}_{\mathrm{s} *} \cdot \mathbf{k}= & \Omega \delta t \frac{k_{2} G m M_{*} R_{\mathrm{p}}^{5}}{a^{3} a_{p}^{3}}\left[-\frac{3}{4} \cos i \sin i \sin \gamma \cos (\Phi-\Psi)\right],
\end{aligned}
$$

and

$$
\begin{aligned}
\mathbf{T}_{* \mathrm{~s}} \cdot \mathbf{s}= & \Omega \delta t \frac{k_{2} G m M_{*} R_{\mathrm{p}}^{5}}{a^{3} a_{p}^{3}}\left[\frac{3}{8} \sin ^{2} i \sin ^{2} \gamma \cos 2(\Phi-\Psi)-\frac{9}{8} \sin ^{2} i \sin ^{2} \gamma\right. \\
& \left.-\frac{3}{4} \cos \gamma \sin \gamma \cos i \sin i \cos (\Phi-\Psi)+\frac{3}{4} \sin ^{2} \gamma\right] \\
\mathbf{T}_{* \mathrm{~s}} \cdot \mathbf{n}= & \Omega \delta t \frac{k_{2} G m M_{*} R_{\mathrm{p}}^{5}}{a^{3} a_{p}^{3}}\left[\frac{3}{4} \cos (\Phi-\Psi) \sin i \sin \gamma \cos ^{2} i-\frac{3}{4} \cos \gamma \cos i \sin ^{2} i\right], \\
\mathbf{T}_{* \mathrm{~s}} \cdot \mathbf{k}= & 0 .
\end{aligned}
$$




\section{References}

[1] Abe, Y., A. Numaguti, G. Komatsu, and Y. Kobayashi 2005. Four climate regimes on a land planet with wet surface: Effects of obliquity change and implications for ancient Mars. Icarus 178, 27-39.

[2] Atobe, K., S. Ida, and T. Ito 2004. Obliquity variations of terrestrial planet in habitable zones. Icarus 168, 223-236.

[3] Agnor, C. B., R. M. Canup, and H. F. Levison 1999. On the character and consequences of large impacts in the late stage of terrestrial planet formation. Icarus 142, 219-237.

[4] Beaulieu, J. -P., and 18 colleagues, 2006. Discovery of a cool planet of 5.5 Earth masses through gravitational microlensing. Nature 439, 439, 437-440.

[5] Berger, A.L., J. Imbrie, J. Hays, G. Kukla and B. Saltzman eds. 1984. Milankovitch and Climate - Understanding the Response to Astronomical Forcing. D. Reidel, Norwell, Mass.

[6] Berger, A. ed. 1989. Climate and Geo-Science - A Challenge for Science and Society in the 21st Century. Kluwer Academic Publishers, Dordrecht.

[7] Bodenheimer, P. and J. B. Pollack 1986. Calculations of the accretion and evolution of giant planets The effects of solid cores. Icarus 67, 391-408.

[8] Borucki, W. J., and 14 colleagues, 2003. The Kepler Mission: Finding the sizes, orbits and frequencies of Earth-size and larger extrasolar planets. Scientific Frontiers in Research on Extrasolar Planets. In: Deming, D., Seager, S. (Eds.), SP Conference Series, 294, pp. $427-440$.

[9] Butler, R. Paul; 2004. A Neptune-mass planet orbiting the Nearby M dwarf GJ 436. Astrophys. J. 617, 580-588.

[10] Canup, R. M. 2004. Dynamics of lunar formation. Ann. Rev. A $\mathscr{E} A$ 42, 441-475.

[11] Chambers, J. E. 2001. Making more terrestrial planets. Icarus 152, 205-224.

[12] Chambers, J. E., G. W. Wetherill, and A. P. Boss 1996. The stability of multi-planet systems. Icarus 119, 261-268.

[13] Colombo, G. 1965. Rotational period of the planet Mercury. Nature 208, 575.

[14] Colombo, G., and I. I. Shapiro 1965. The rotation of the planet Mercury. SAO Special Report no. 188R.

[15] Correia, A. C. M., and J. Laskar 2001. The four final rotation states of Venus. Nature 411, 767-770.

[16] Counselman, C.C., III. 1973. Outcomes of Tidal Evolution. Astrophys. J. 180, 307-316.

[17] Darwin, G. H. 1879. A tidal theory of the evolution of satellites. The Observatory 3, 79-84. 
[18] Gold, T. and S. Soter 1969. Atmospheric tides and the resonant rotation of Venus. Icarus 11, 356-366.

[19] Goldreich, P. 1966. History of the lunar orbit. Rev. Grophys. 4, 411-439.

[20] Goldreich, P. and S. J. Peale 1966. Spin-orbit coupling in the solar system. Astron. J. 71, 425-438.

[21] Goldreich, P. and S. J. Peale 1970. The obliquity of Venus. Astron. J. 75, 273-284.

[22] Hayashi, C., K. Nakazawa, and Y. Nakagawa 1985. Formation of the solar system. In: Protostars and planets II, Tucson, AZ, University of Arizona Press, 1985, pp. 1100-1153.

[23] Stevenson, D. J. 1987. Origin of the moon-the collision hypothesis, Ann. Rev. Earth Planet Sci. 15, 271-315.

[24] Ida, S., R. M. Canup, and G. R. Stewart 1997. Lunar accretion from an impact generated disk. Nature 389, 353-357.

[25] Ida, S. and D. N. C. Lin 2004. Toward a deterministic model of planetary formation. II. The formation and retention of gas giant planets around stars with a range of metallicities. Astrophys. J. 616, 567-572.

[26] Iwasaki, K., H. Emori, K. Nakazawa, and H. Tanaka 2002. Orbital stability of a protoplanet system under a drag force proportional to the random velocity. PASJ 54, 471-479.

[27] Kokubo, E., and S. Ida 1998. Oligarchic growth of protoplanets. Icarus 131, 171-178.

[28] Kokubo, E., and S. Ida 2000. Formation of protoplanets from planetesimals in the solar nebula. Icarus 143, 15-27.

[29] Kokubo, E., J. Makino, and S. Ida 2000. Evolution of a circumterrestrial disk and formation of a single Moon. Icarus 148, 419-436.

[30] Laskar, J. 1996. Large scale chaos and marginal stability in the solar system. Celest. Mech. Dyn. Astron. 64, 115-162.

[31] Laskar, J. and P. Robutel 1993. The chaotic obliquity of the planets. Nature 361, 608-612.

[32] Laskar, J., F. Joutel, and P. Robutel 1993. Stabilization of the earth's obliquity by the moon. Nature 361, 615-617.

[33] Lissauer, J. J., L. Dones, and K. Ohtsuki 2000. Origin and evolution of terrestrial planet rotation. In Origin of Earth and Moon (R. M. Canup, and K. Righter, Eds.), pp. 101-112. Univ. of Arizona Press, Tucson.

[34] Lovis, C. J. et al. 2006. An extrasolar planetary system with three Neptune-mass planets. Nature 441, 305-309.

[35] Mayor, M. and D. Queloz 1995. A jupiter-mass companion to a solar-type star. Nature 378, 355-359. 
[36] Milankovitch, M. 1941. Kanon der Erdbestrahlung und seine Anwendung auf das Eiszeitproblem. KanonKöniglich Serbische Academie Publication 133, Königlich Serbische Academie.

[37] Mignard, F. 1979. The evolution of the lunar orbit revisited. I. Moon Planets 20, 301-315.

[38] Mignard, F. 1980. The evolution of the lunar orbit revisited. II. Moon Planets 23, 185-201.

[39] Mignard, F. 1981. The lunar orbit revisited. III. Moon Planets 23, 185-201.

[40] Mizuno, H. 1980. Formation of the giant planets. Pro. Theor. Phys. 64, 544-557.

[41] Munk, W. H., and G. J. F. MacDonald 1960. Continentality and the gravitational field of the Earth. J. Geophys. Res. 65, 2169.

[42] Nérson de Surgy, O. 1996. Influence des effects dissipatifs sur les variations á long terme des obliquités planétaires. Observatoire de Paris.

[43] Rivera, E. J. 2005. A $7.5 M_{\oplus}$ Planet Orbiting the Nearby Star, GJ 876. Astrophys. J. 634, 625-640.

[44] Ruden, S. P. 1999. The formation of planets. In: Lada, C. J. and N. D. Kylafis (Eds.), The Origin of Stars and Planetary Systems, Kluwer Academic Publishers, pp. 643.

[45] Touma, J., and J. Wisdom 1994. Evolution of the Earth-Moon system. Astron. J. 108, 1943-1961.

[46] Ward, W. R. 1973. Large-scale variations in the obliquity of Mars. Science 181, 260-262.

[47] Ward, W. R. 1974. Climate variations on Mars 1. Astronomical theory of insolation. J. Geophys. Res. 79, 3375-3386.

[48] Ward, W. R. 1979. Present obliquity oscillations of Mars - Fourth-order accuracy in orbital E and I. J. Geophys. Res. 84, 237-241.

[49] Ward, W. R. and M. L. Reid 1973. Solar tidal friction and satellite loss. Mon. Not. R. astr. Soc. 164, 21-32.

[50] Williams, D. M. and J. L. Kasting 1997. Habitable planets with high obliquities. Icarus 129, 254-267.

[51] Yoder, C. F. 1997. Venusian spin dynamics, In: Bougher, s. W., D. M. Hunten, R. J. Philips. (Eds.), Venus II : Geology, Geophysics, Atmosphere, and Solar Wind Environment, University of Arizona Press, Tucson, pp. 1087-1124. 


\section{Figure captions}

Figure 1 The tidal evolution of the Earth-Moon system: (a) the obliquity of the Earth to the ecliptic $\gamma$, (b) the inclination of the Moon's orbit to the ecliptic $i$, (c) that to the Earth's equator $\epsilon$. The ranges of oscillation during precession are indicated by gray patches.

Figure 2 Tidal deformation and phase lag are illustrated. The planet (represented by the circle in dashed line) is distorted (oval in thick solid line) by the gravitational force of the external body (the small circle in thick solid line). The tidal friction causes a phase shift $(\delta)$ in the response of a planet. The case in which the planetary spin is faster than the external body's orbital motion is shown.

Figure 3 The geometry of the orbital plane of the planet, its equatorial plane, and the orbital plane of the satellite is shown. Their unit normal vectors are $\mathbf{s}, \mathbf{k}$, and $\mathbf{n}$. The obliquity $\gamma$, the inclinations $i$ and $\epsilon$ are indicated.

Figure 4 The trajectories of the tips of $\mathbf{s}$ and $\mathbf{n}$ projected onto the orbital plane $(X-Y)$ of the planet in the case of the Earth-Moon system. (a) $a \simeq 5 R_{\oplus}$, (b) $a \simeq 15 R_{\oplus}$, (c) $a \simeq 60 R_{\oplus}$ (the present configuration). The solid and dashed lines show trajectories of $\mathbf{s}$ and $\mathbf{n}$, respectively. Filled squares and triangles indicate the positions of $\mathbf{s}$ and $\mathbf{n}$. They move on the trajectories in labeled number order.

Figure 5 The numerical calculated tidal evolution of the system with $m=0.02 M_{\mathrm{p}}, \gamma_{0}=10^{\circ}$ : (a) the obliquity $\gamma,(\mathrm{b})$ the inclination of the satellite orbit to the planetary orbit $i$, (c) that to the planet's equator $\epsilon$, and (d) the semi-major axis of the satellite $a$ (dashed line), the inner co-rotation radius $a_{\mathrm{c}, \text { in }}$ (gray solid line) and the outer one $a_{\mathrm{c}, \text { out }}$ (black solid line) calculated by $L_{Z 0}=L_{Z \mathrm{c}}$ (Eqs. 23 and 25). In the panels (a), (b), and (c), the ranges of oscillation during precession are indicated by gray patches.

Figure 6] Same as Fig. 5, but $m=0.01 M_{\mathrm{p}}, \gamma_{0}=80^{\circ}$.

Figure 7 Schematic illustration of the precession motion of $\mathbf{s}$ and $\mathbf{n}$. When $a \lesssim a_{\text {crit }}$, $\mathbf{s}$ and $\mathbf{n}$ precess about their total vector (type I precession). During the precession, the mutual inclination $(\epsilon)$ between $\mathbf{s}$ and $\mathbf{n}$ is almost constant. When $a \gtrsim a_{\text {crit }}, \mathbf{s}$ and $\mathbf{n}$ precess about $\mathbf{k}$, respectively (type III precession). During the precession, $\epsilon$ oscillates from $0^{\circ}$ to $\sim 2 \gamma$.

Figure 8 Same as Fig. [5, but $m=0.04 M_{\mathrm{p}}, \gamma_{0}=40^{\circ}$. 
Figure 9 The dependence of tidal evolution on initial conditions (initial obliquity $\gamma_{0}$ and satellite-planet mass ratio $m / M_{\mathrm{p}}$ ) found by the numerical integration. Type A1 evolution with $\gamma \rightarrow 0^{\circ}\left(\right.$ for $\gamma_{0}<90^{\circ}$ ) or $\gamma \rightarrow 180^{\circ}$ (for $\gamma_{0}>90^{\circ}$ ) is plotted by open circles. A3 evolution which shows $\gamma \rightarrow 0^{\circ}$ with $\gamma_{0}>90^{\circ}$ is plotted by filled squares. Crosses and filled circles represent B and C evolution, respectively. Triangles represent A2 evolution.

Figure 10 Contours of angular momentum $\tilde{L}$ (solid lines) and energy $\tilde{E}$ (dashed lines) in a planar system. Planetary spin and satellite's orbital periods are equal when $\tilde{\Omega}=\tilde{n}^{3}$, represented by the dotted line. Critical points are shown by filled triangles, at which all lines of constant- $\tilde{L}$, constant- $\tilde{E}$, and synchronism are mutually tangent. Path of tidal evolution in the $(\tilde{\Omega}, \tilde{n})$-plane are indicated by arrows.

Figure 11 The time evolution of the normalized total angular momentum $(\tilde{L})$ and its $Z$ component $\left(\tilde{L_{\mathrm{Z}}}\right)$, and the total mechanical energy of the planet-satellite system $(\tilde{E})$, in the cases of (a) $m=0.02 M_{\mathrm{p}}$ and $\gamma_{0}=10^{\circ},(\mathrm{b}) m=0.01 M_{\mathrm{p}}$ and $\gamma_{0}=80^{\circ}$ and $(\mathrm{c}) m=0.04 M_{\mathrm{p}}$ and $\gamma_{0}=40^{\circ}$.

Figure 12 Path of the tidal evolution of non-planar systems in the case of (a) $m=0.02 M_{\mathrm{p}}$ and $\gamma_{0}=10^{\circ}$, (b) $m=0.01 M_{\mathrm{p}}$ and $\gamma_{0}=80^{\circ}$, (c) $m=0.04 M_{\mathrm{p}}$ and $\gamma_{0}=40^{\circ}$. Thick solid lines are the numerical results. Solid and dashed lines are contours of $\tilde{L}$ lines assuming $\epsilon=0$ and $\tilde{E}$. Spin-orbit synchronism $\left(\tilde{\Omega} \cos \epsilon=\tilde{n}^{3}\right)$ is represented by the dotted lines.

Figure 13 Qualitatively different tidal evolution predicted by the theoretical arguments, as a function of $\left(m / M_{\mathrm{p}}, \gamma_{0}\right)$. B and $\mathrm{C}$ are separated by $\left|\tilde{L}_{Z}\right| \simeq \tilde{L}^{*}$ (thick dashed lines). A1 and $\mathrm{B}$ are separated by $a_{\mathrm{crit}} \simeq a_{\mathrm{c}, \text { out }}^{\infty}$ (thick solid line). A2 and B/C are separated by the vertical line which has the mass determined by $a_{\text {crit }} \simeq a_{\mathrm{c}, \text { out }}^{\infty}$ with $\gamma_{0}=0^{\circ}$. The boundary between $\mathrm{A} 1$ and $\mathrm{A} 3$ is given by $\Delta t_{\mathrm{A} 3}=\Delta t_{\mathrm{A} 1}$ (thin solid lines).

Figure 14 Comparison of the analytically estimated boundaries with the numerical results in Fig. 9. For the analytical estimate, see text.

Figure 15 Schematic illustration of typical obliquity changes in individual evolution types.

Figure 16 The timescales of the tidal evolution. with $Q=12$ and $k_{2}=0.30$, using Eqs.(30). The contours of the timescales are represented by dotted lines. From right to left, contours represent $10^{5}, 10^{6}, \ldots, 10^{12}$ yrs. 

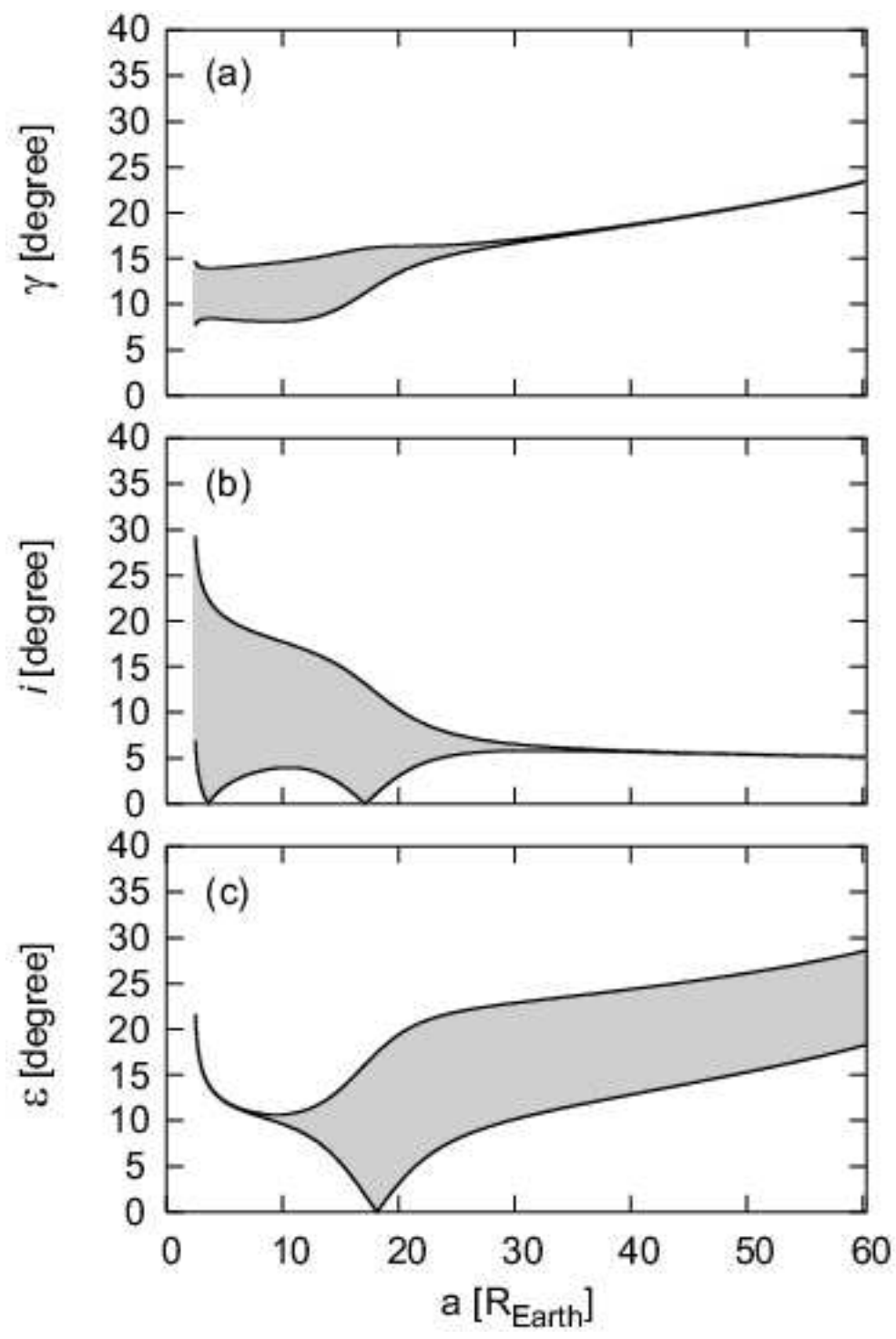

Figure 1: Atobe and Ida 


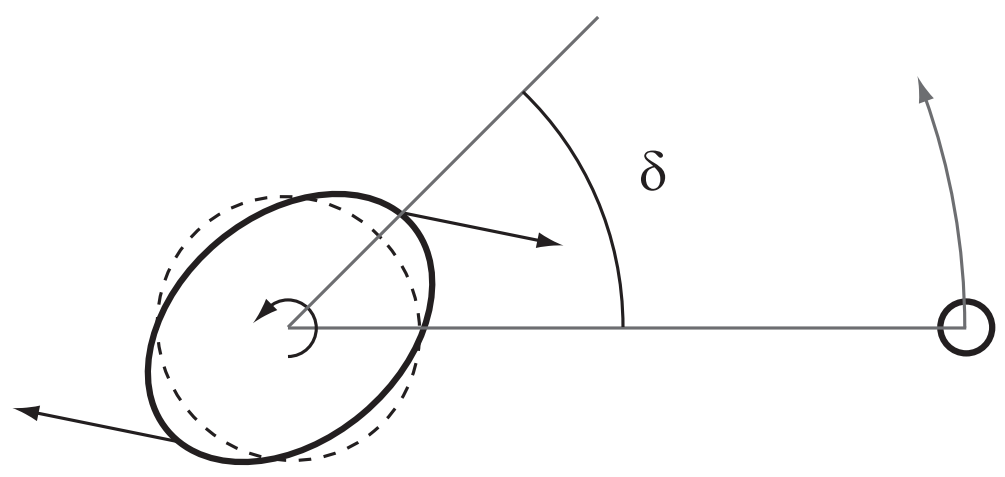

Figure 2: Atobe and Ida 


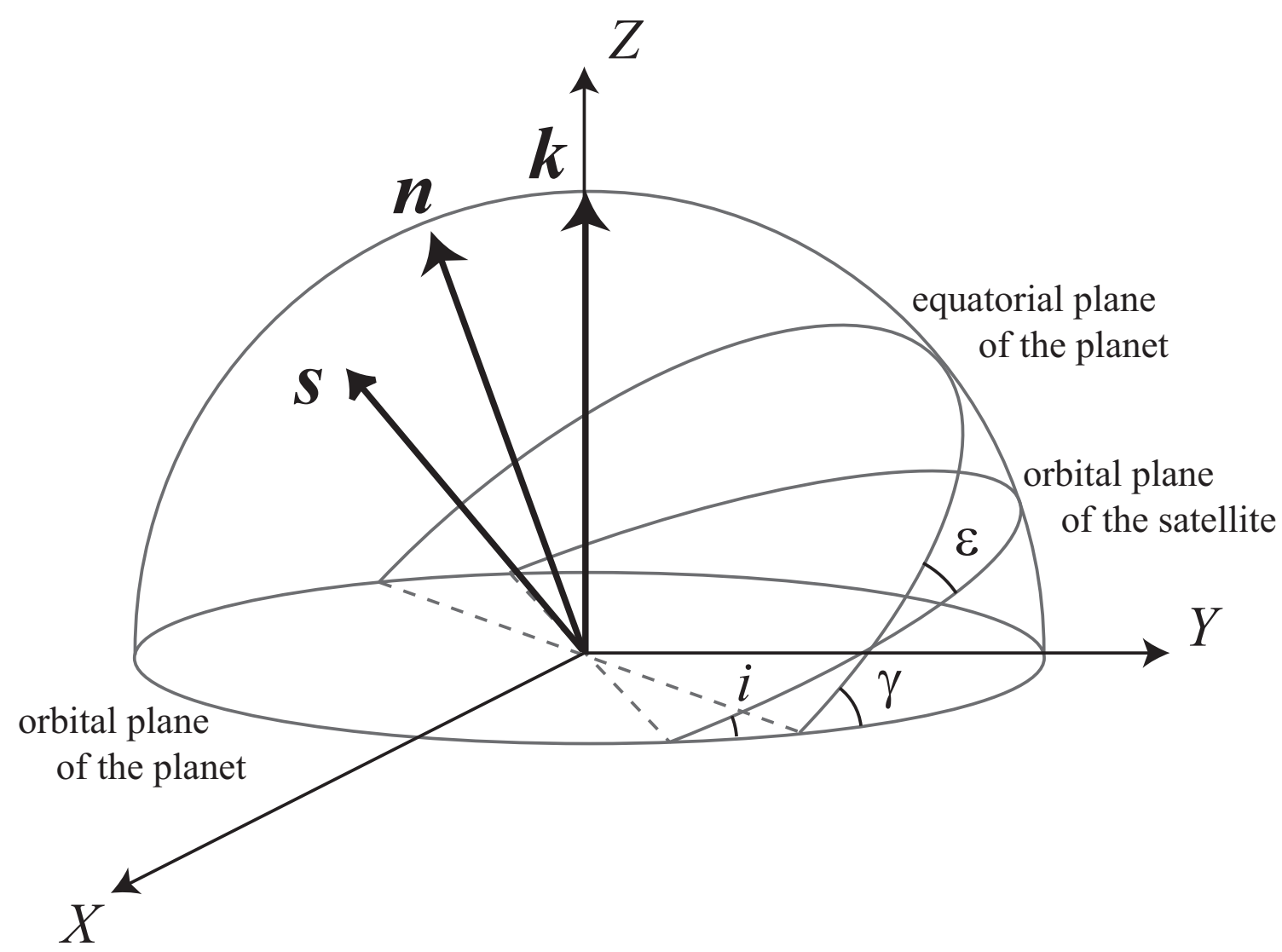

Figure 3: Atobe and Ida 

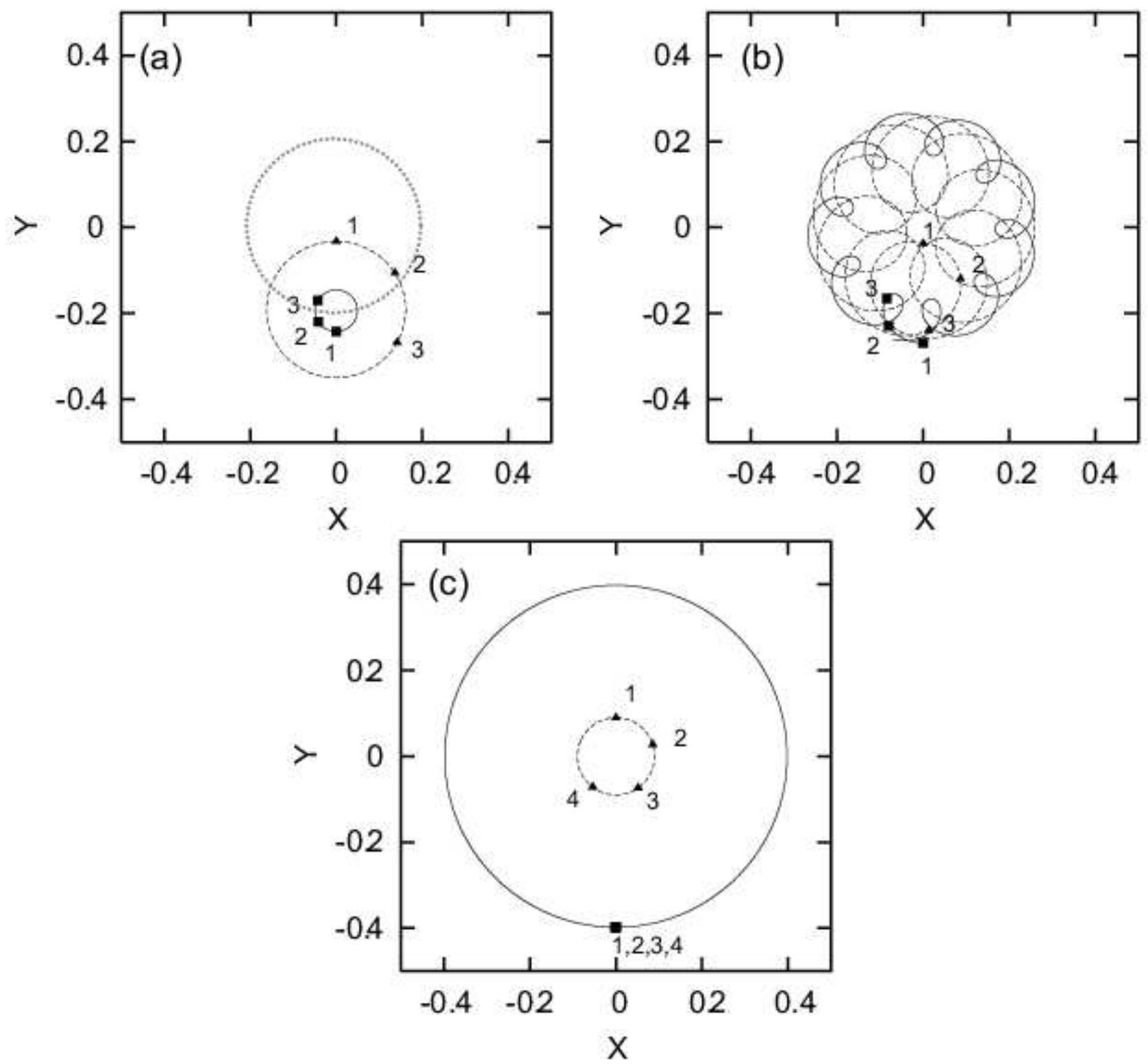

Figure 4: Atobe and Ida 

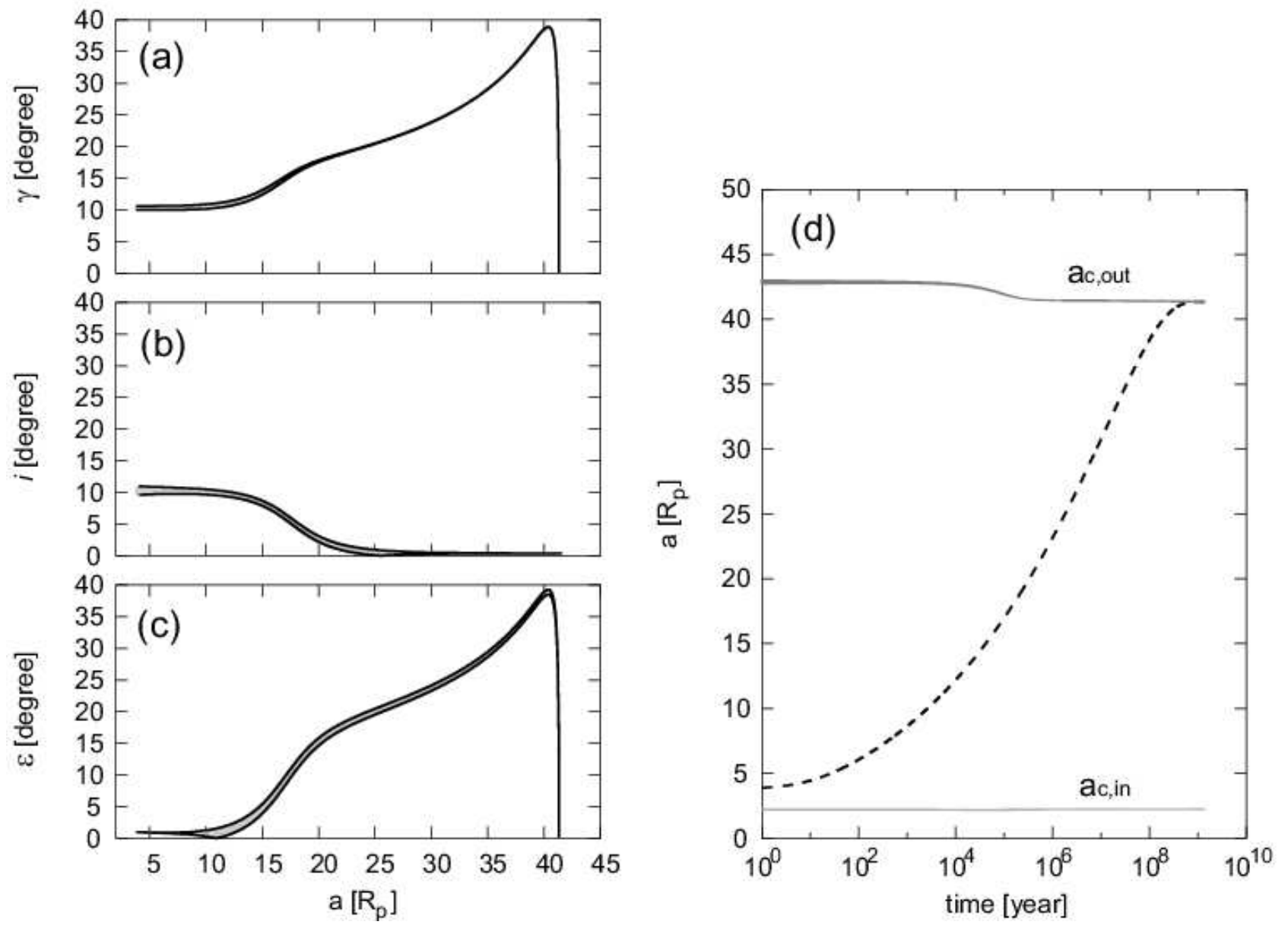

Figure 5: Atobe and Ida 

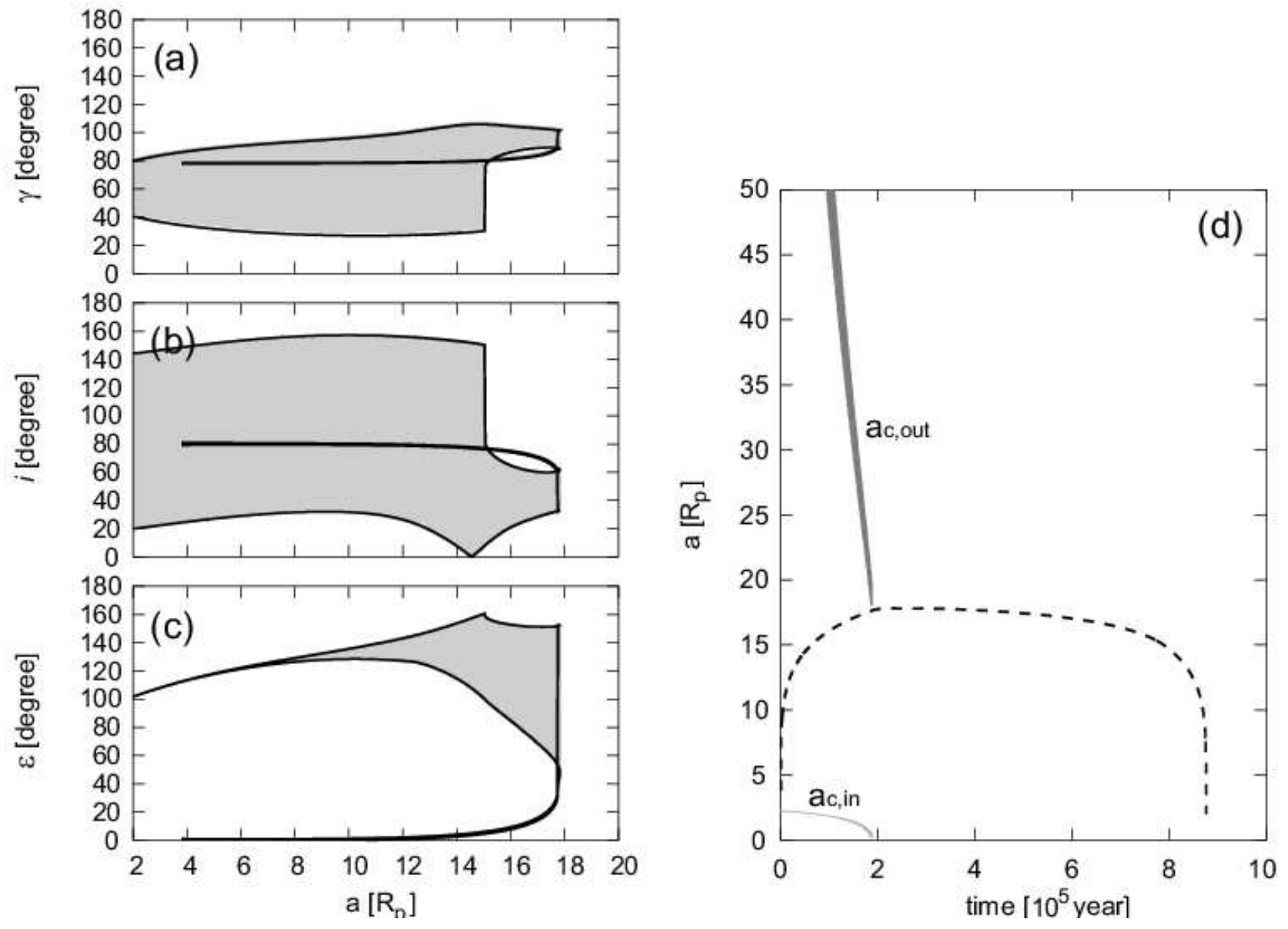

Figure 6: Atobe and Ida 

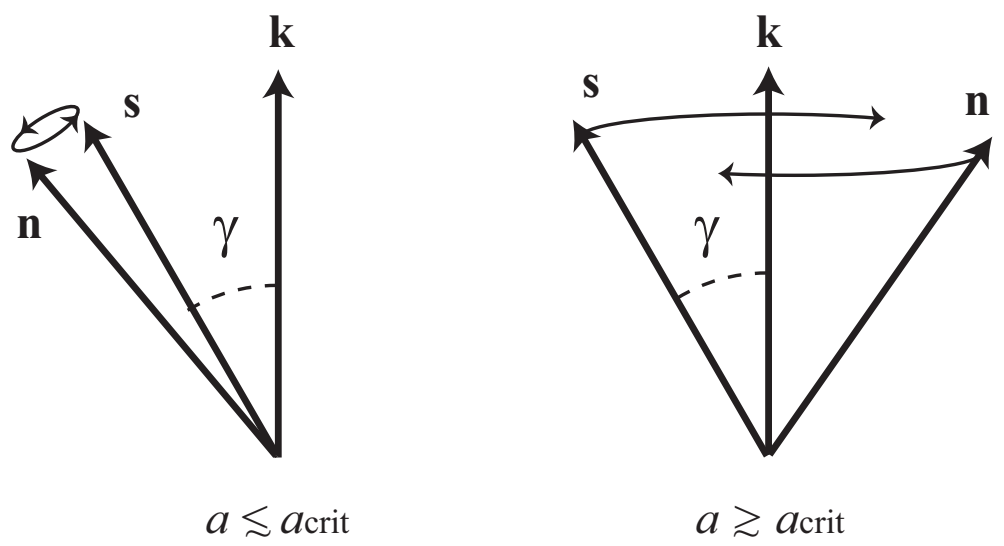

Figure 7: Atobe and Ida 

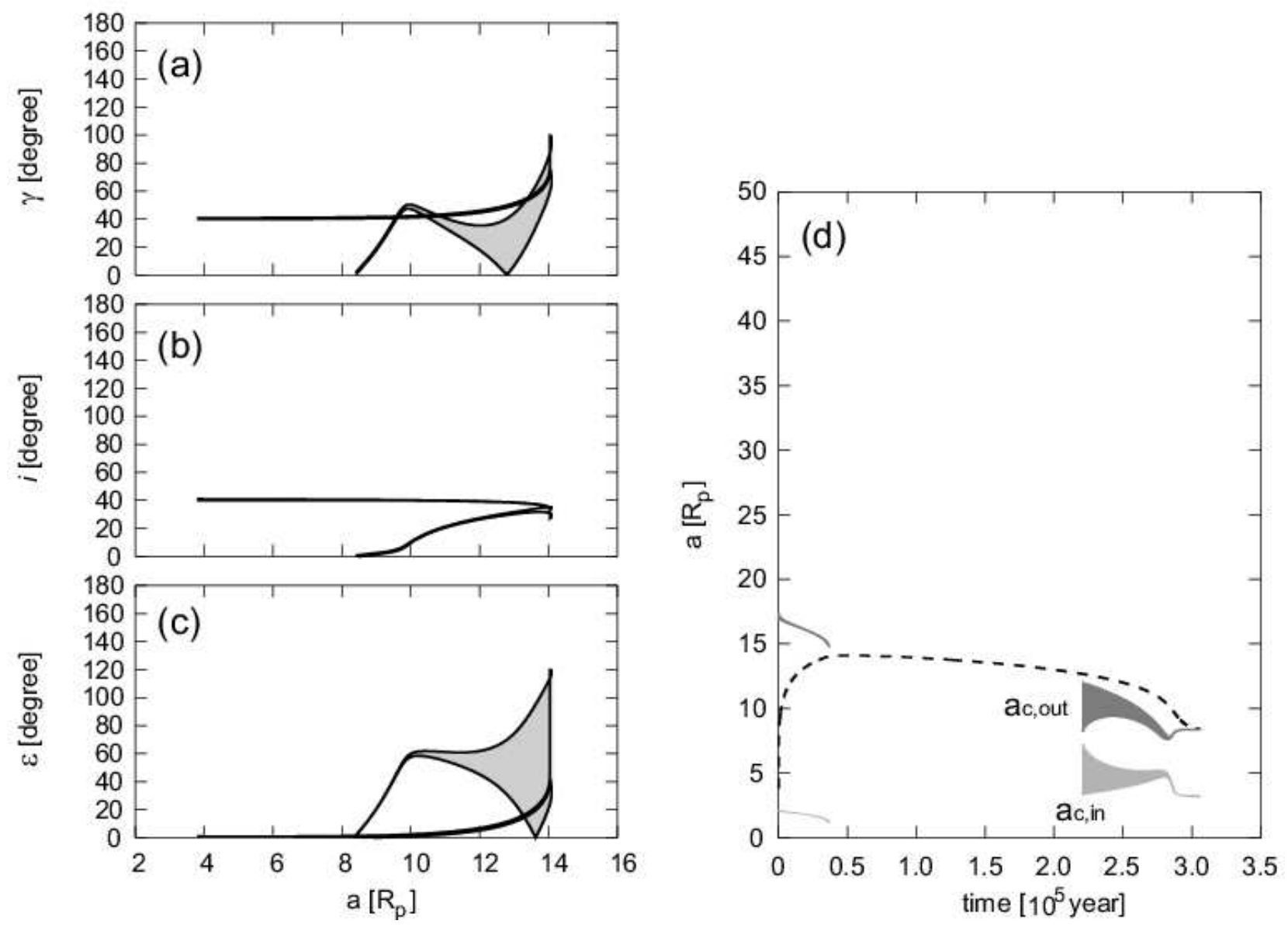

Figure 8: Atobe and Ida 


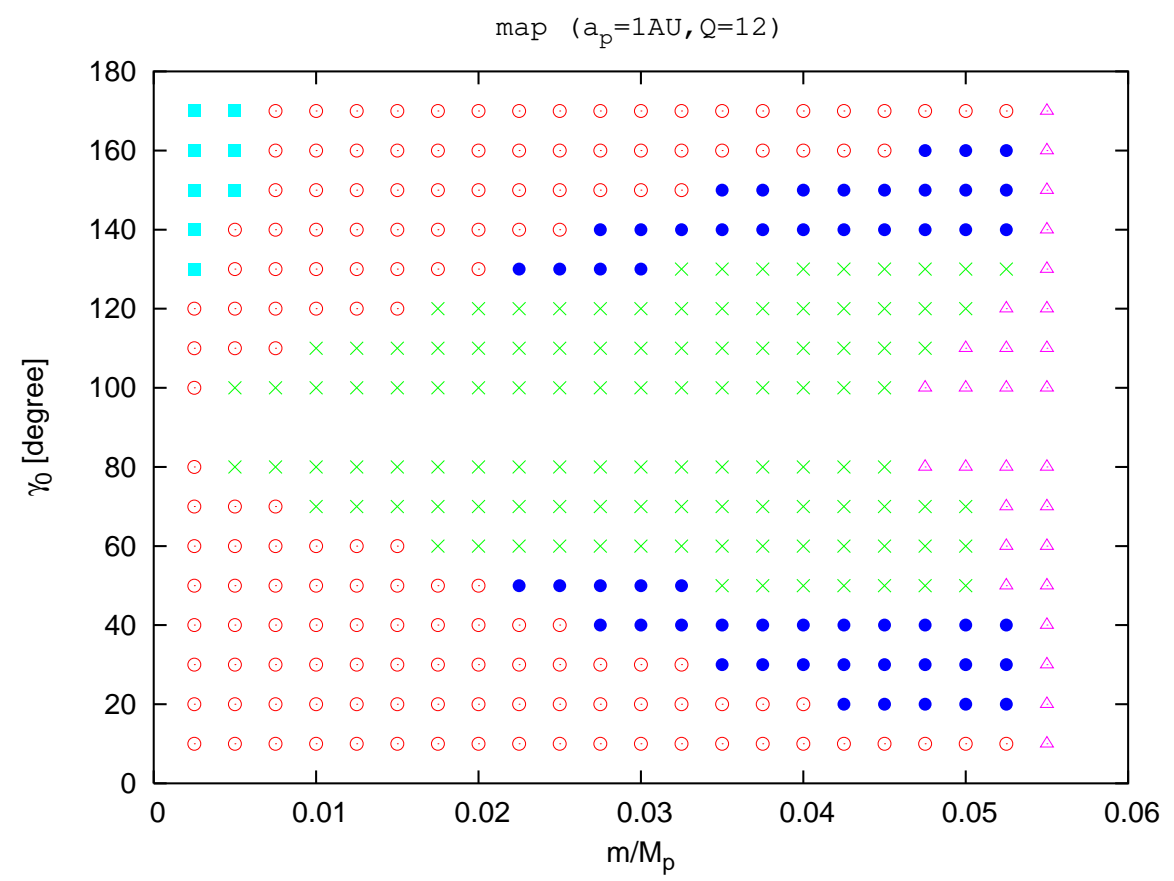

Figure 9: Atobe and Ida 


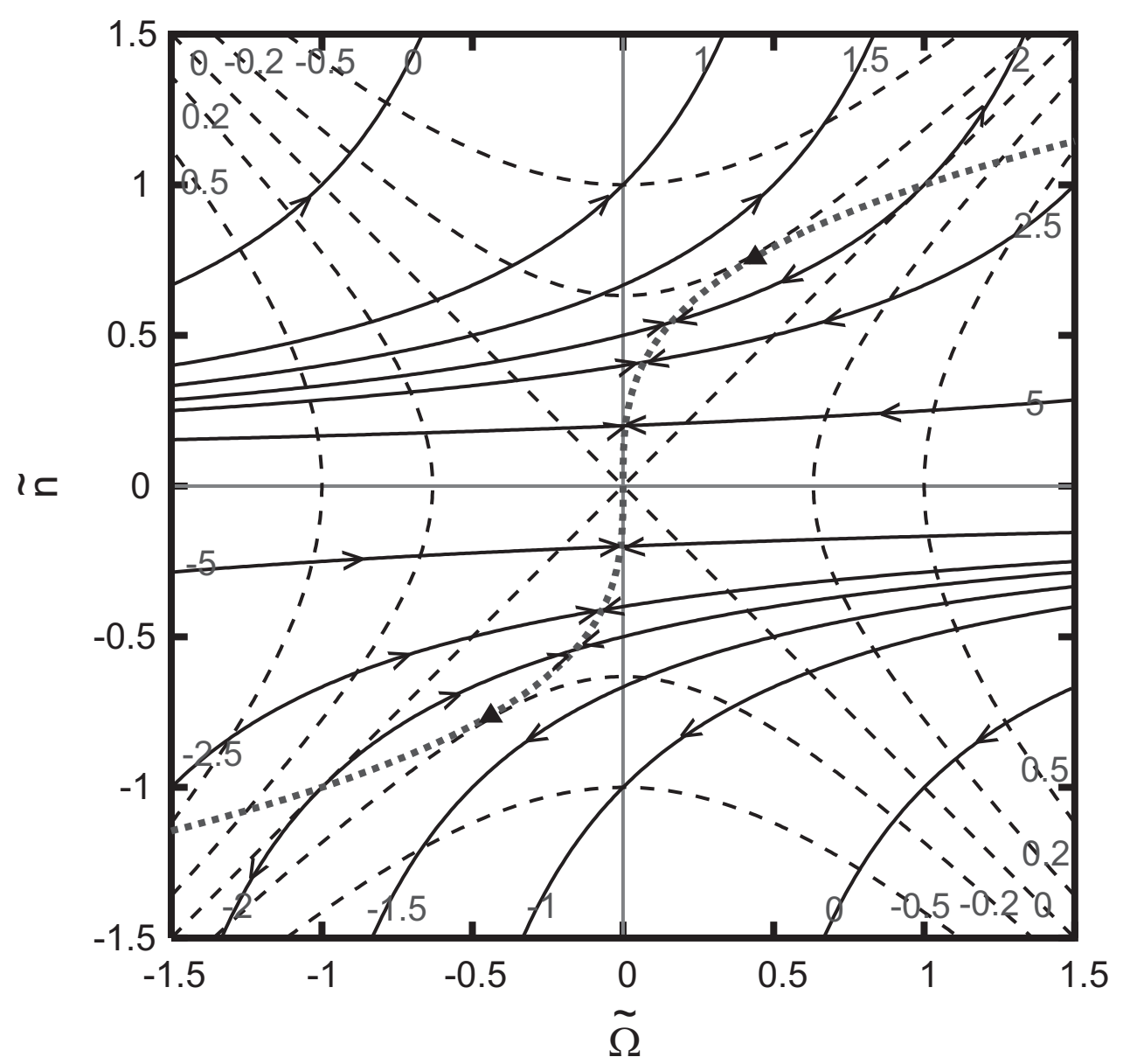

Figure 10: Atobe and Ida 
(a)

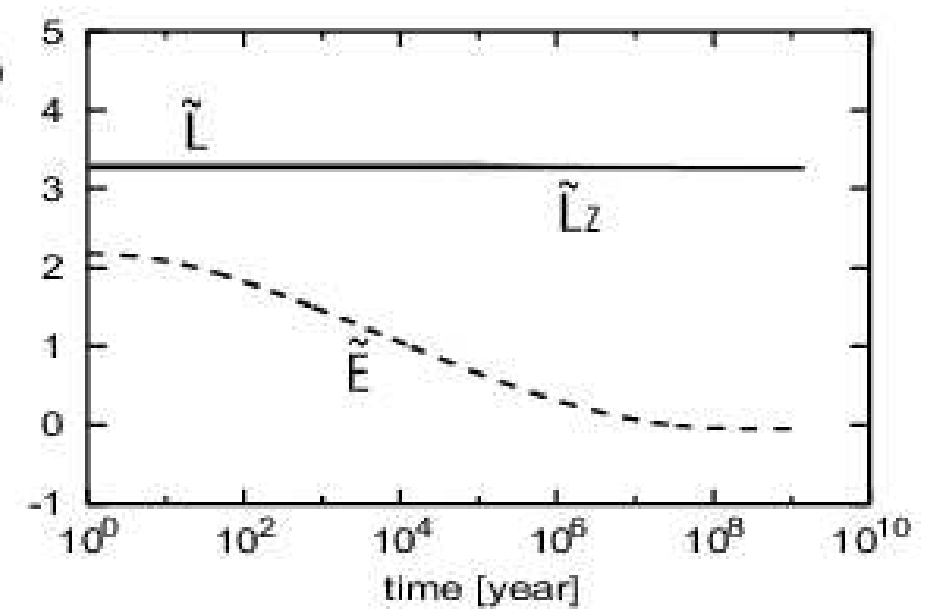

(b)

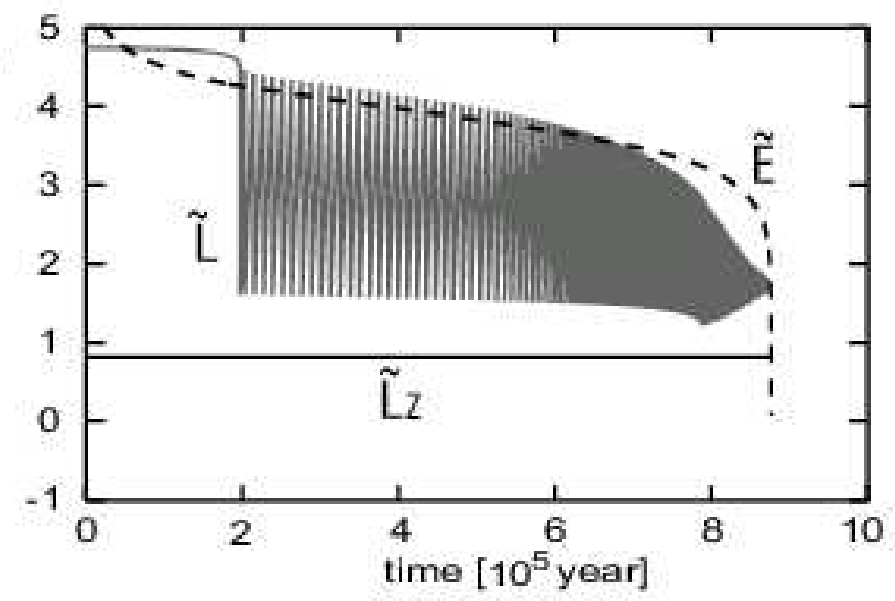

(c)

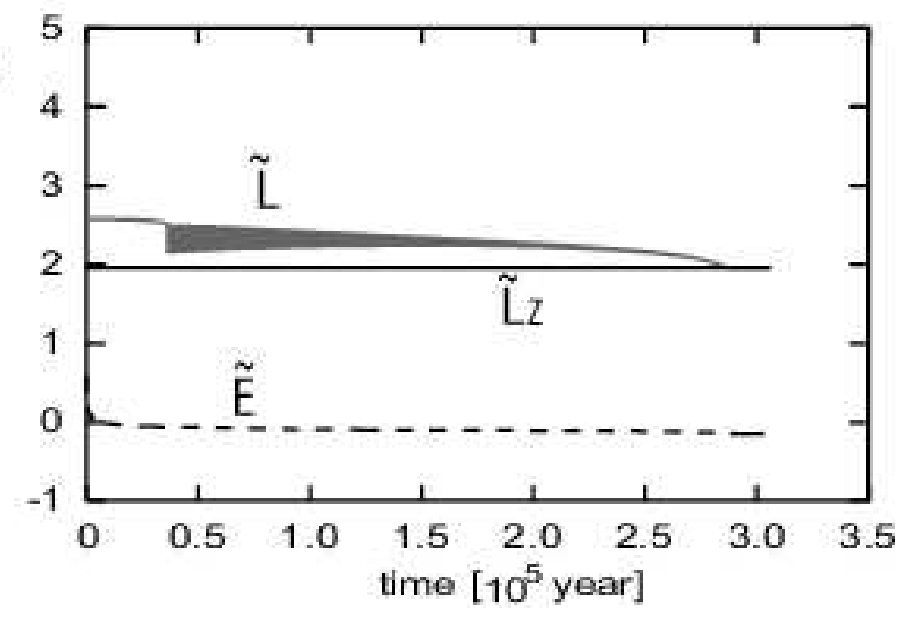

Figure 11: Atobe and Ida 

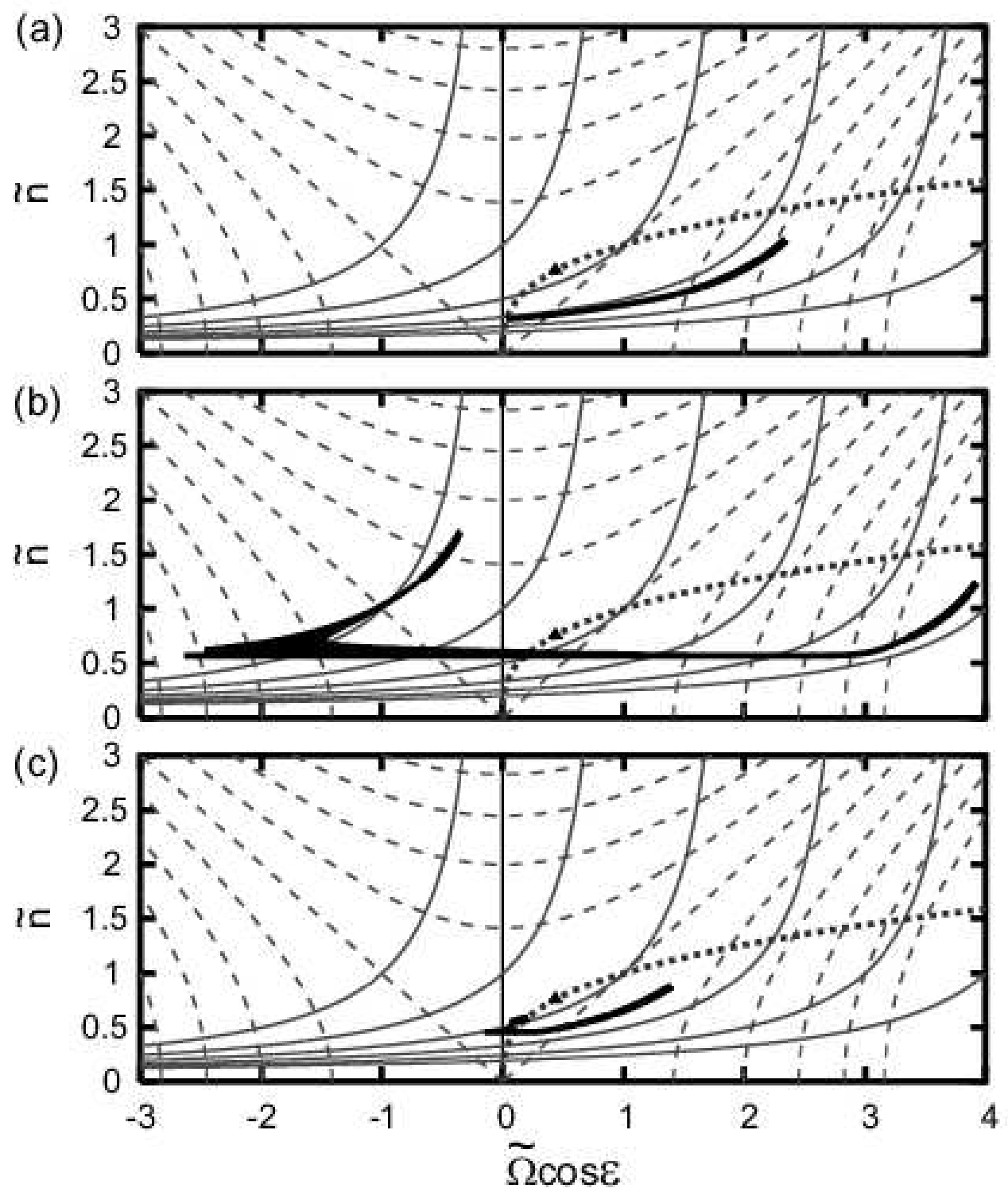

Figure 12: Atobe and Ida 


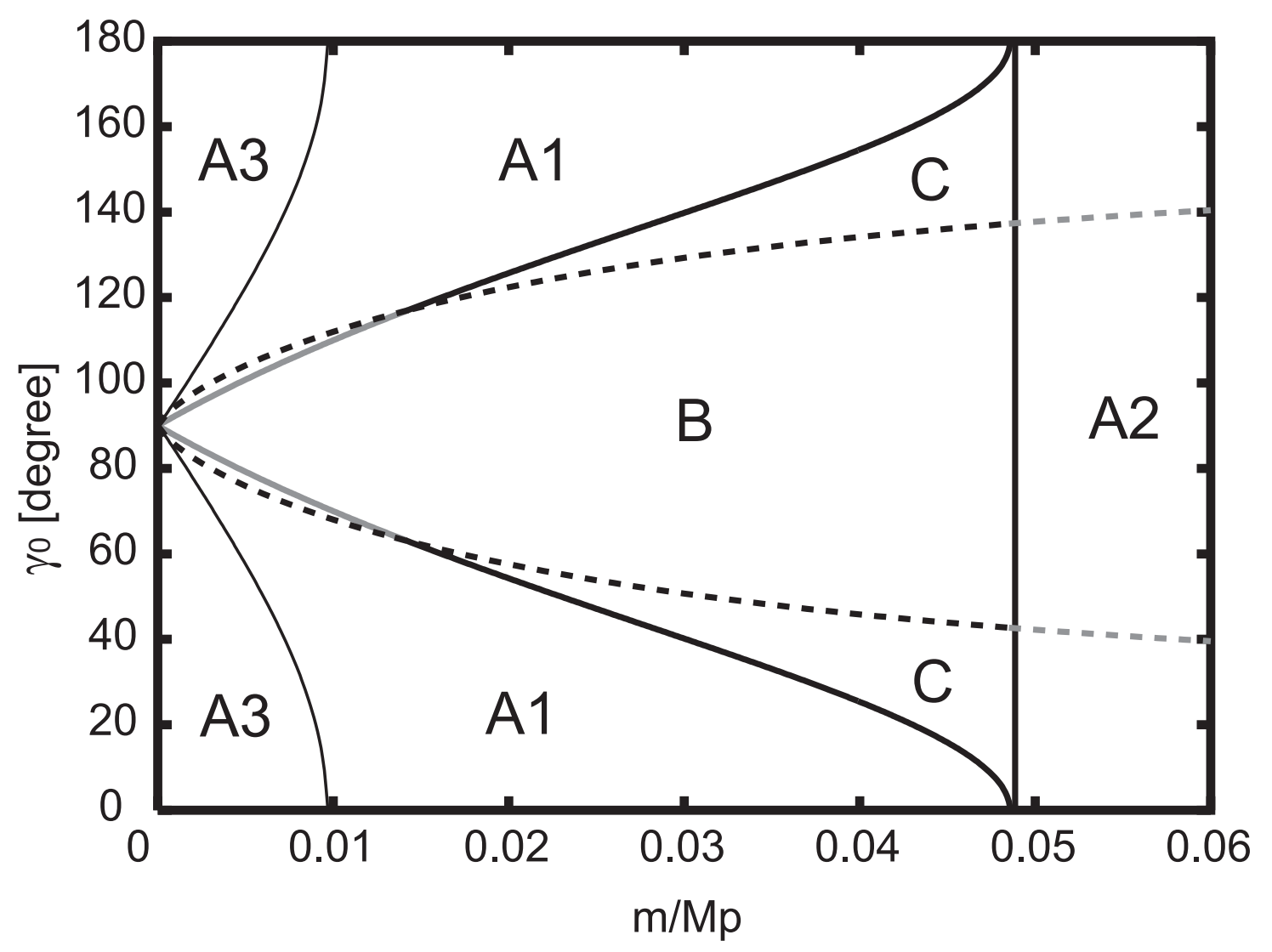

Figure 13: Atobe and Ida 


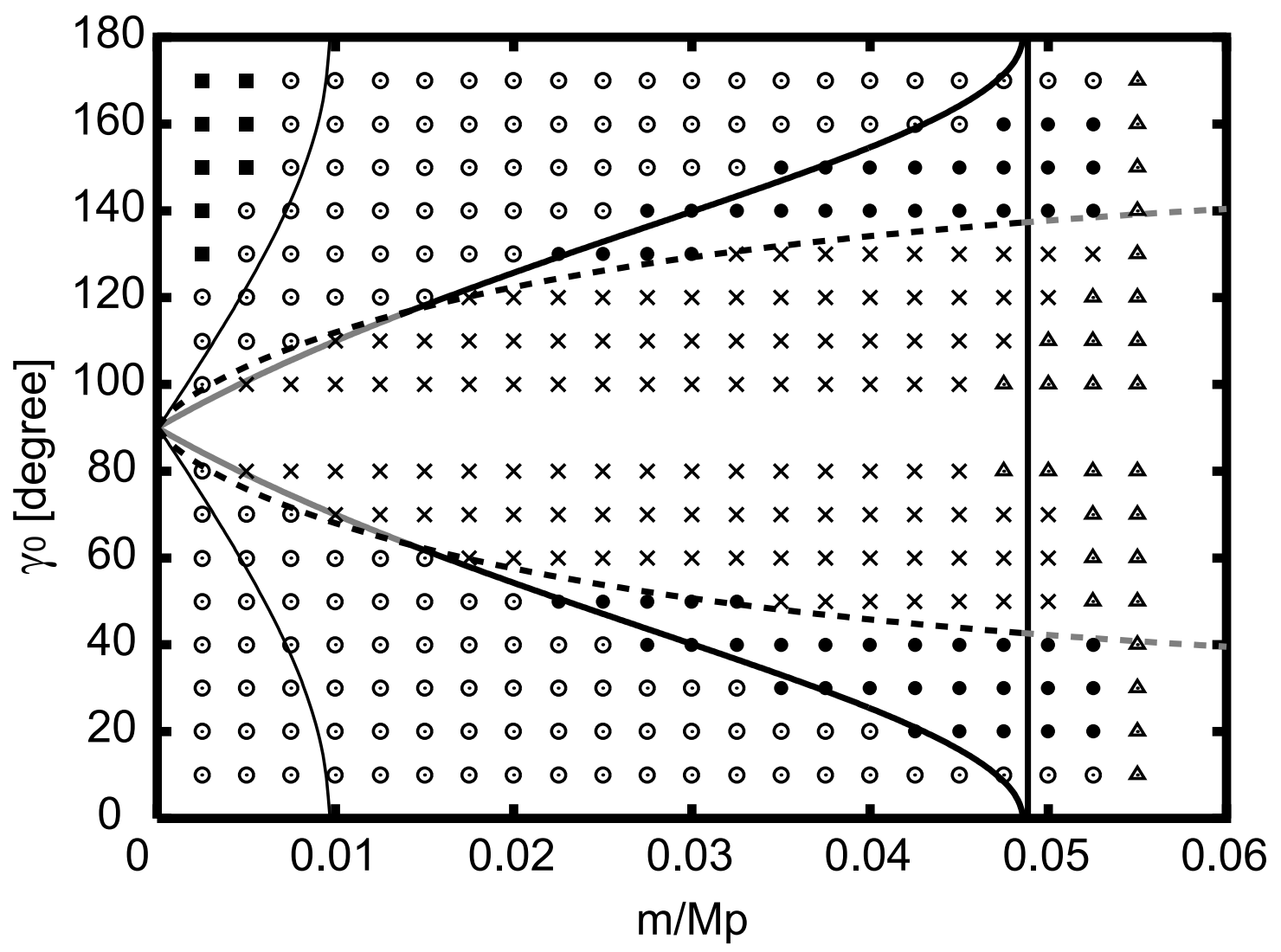

Figure 14: Atobe and Ida 


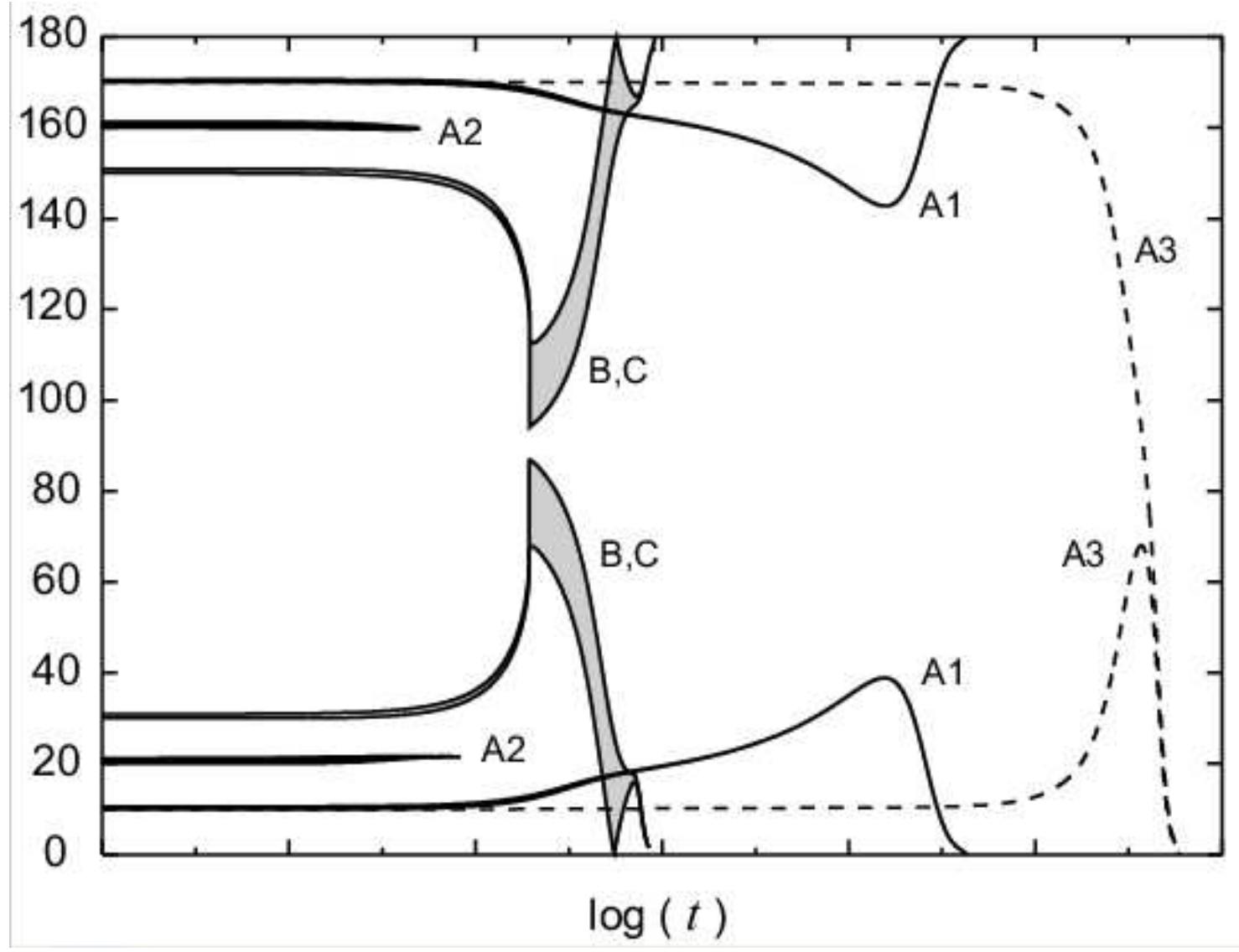

Figure 15: Atobe and Ida 


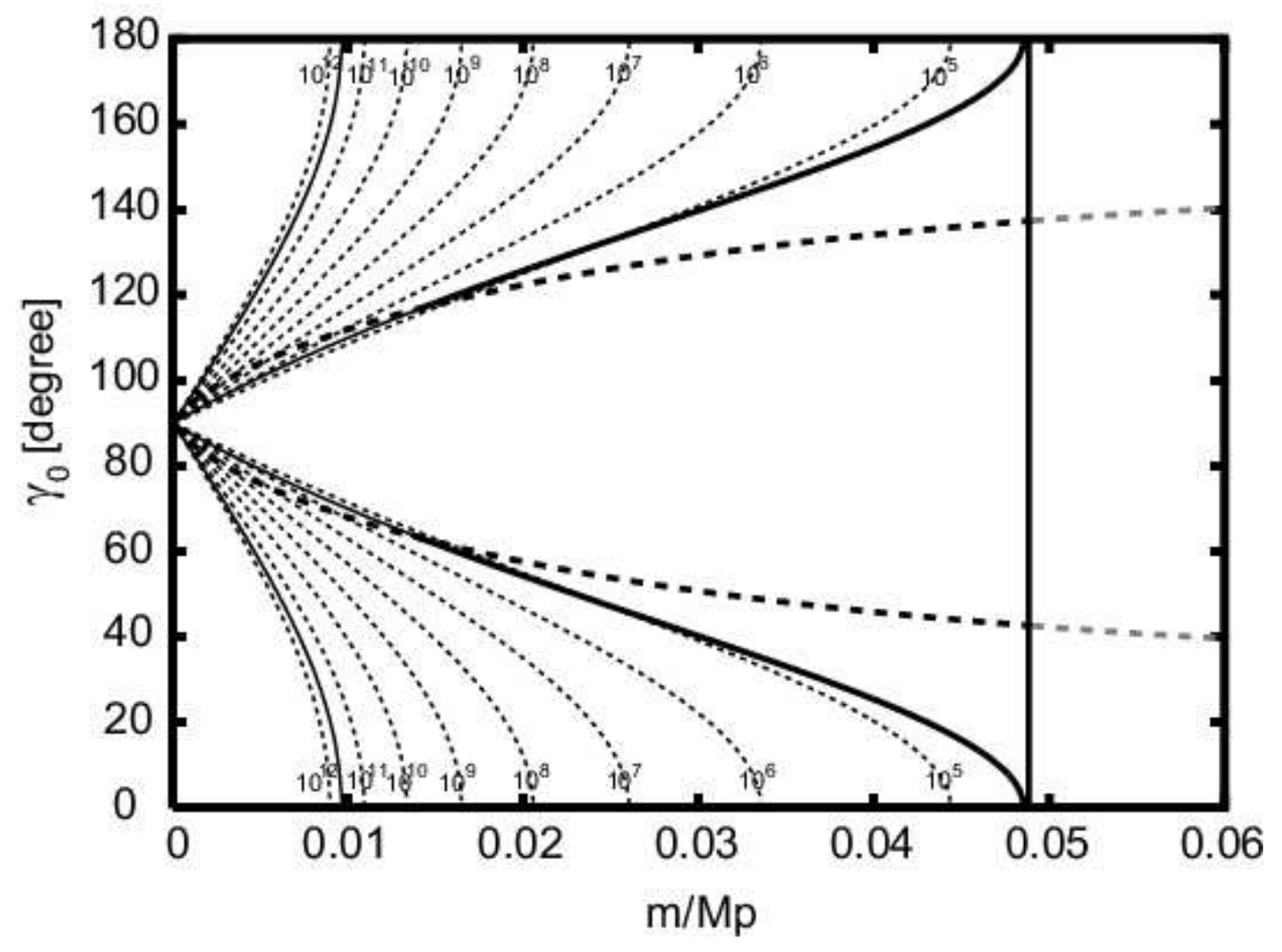

Figure 16: Atobe and Ida 